\title{
A variant of 3-3-1 model for the generation of the SM fermion mass and mixing pattern
}

\author{
A.E. Cárcamo Hernández, ${ }^{a}$ Sergey Kovalenko, ${ }^{a}$ H. N. Long ${ }^{b, c, 1}$ and Ivan Schmidt ${ }^{a}$ \\ ${ }^{a}$ Universidad Técnica Federico Santa María and Centro Científico-Tecnológico de Valparaíso, \\ Casilla 110-V, Valparaíso, Chile \\ ${ }^{b}$ Theoretical Particle Physics and Cosmology Research Group, \\ Advanced Institute for Materials Science, Ton Duc Thang University, \\ Ho Chi Minh City, Vietnam \\ ${ }^{c}$ Faculty of Applied Sciences, Ton Duc Thang University, \\ Ho Chi Minh City, Vietnam \\ E-mail: antonio.carcamo@usm.cl, sergey.kovalenko@usm.cl, \\ hoangngoclong@tdt.edu.vn, ivan.schmidt@usm.cl
}

ABSTRACT: We propose an extension of the 3-3-1 model with an additional symmetry group $Z_{2} \times Z_{4} \times \mathrm{U}(1)_{L_{g}}$ and an extended scalar sector. To our best knowledge this is the first example of a renormalizable 3-3-1 model, which allows explanation the SM fermion mass hierarchy by a sequential loop suppression: tree-level top and exotic fermion masses, 1-loop bottom, charm, tau and muon masses; 2-loop masses for the light up, down, strange quarks as well as for the electron. The light active neutrino masses are generated from a combination of linear and inverse seesaw mechanisms at two loop level. The model also has viable fermionic and scalar dark matter candidates.

Keywords: Beyond Standard Model, Discrete Symmetries, Gauge Symmetry, Quark Masses and SM Parameters

ARXiv EPrint: 1705.09169

\footnotetext{
${ }^{1}$ Corresponding author.
} 


\section{Contents}

1 Introduction 1

2 The model 3

$\begin{array}{ll}2.1 & \text { Tadpole cancellation mechanisms } \\ \end{array}$

3 Quark masses and mixings 14

4 Lepton masses and mixings $\quad 16$

5 Discussions and conclusions $\quad 20$

$\begin{array}{ll}\text { A Generalized lepton number } & 22\end{array}$

B Perturbative diagonalization of the neutrino mass matrix 23

$\begin{array}{ll}\text { C Sterile neutrino mass spectrum } & 25\end{array}$

\section{Introduction}

Despite the great consistency of the Standard Model (SM) with experimental data, recently confirmed by the LHC discovery of the $126 \mathrm{GeV}$ Higgs boson [1, 2], it has several unexplained issues [3]. Among the most pressings ones are the smallness of neutrino masses, the fermion mass and mixing hierarchy, and the existence of three fermion families.

In the SM the flavor structure of the Yukawa interactions is not restricted by gauge invariance. Consequently, fermion masses and mixings are left unfixed, and the SM does not provide an explanation for their large hierarchy, which spreads over a range of five orders of magnitude in the quark sector, and a dramatically broader range of about 11 orders of magnitude, if we include the neutrinos. Even though in the SM these parameters appear only through Yukawa interaction terms and not in explicit mass terms, this mechanism does not provide an explanation for their values, but only translates the problem to fitting different Yukawa couplings, one for each mass and with disparate values for some of them. The origin of quark mixing and the size of $\mathrm{CP}$ violation in this sector is also a related issue. A fundamental theory is expected to provide a dynamical explanation for the masses and mixings.

While the mixing angles in the quark sector are very small, in the lepton sector two of the mixing angles are large, and one mixing angle is small. This suggests a different kind of New Physics for the neutrino sector from the one present in the quark mass and mixing pattern. Experiments with solar, atmospheric and reactor neutrinos have brought clear evidence of neutrino oscillations from the measured non vanishing neutrino mass 
squared splittings. This brings compelling and indubitable evidence that at least two of the neutrinos have non vanishing masses, much smaller, by many orders of magnitude, than the SM charged fermion masses, and that the three neutrino flavors mix with each other.

The flavor puzzle of the SM indicates that New Physics has to be advocated in order to explain the prevailing pattern of fermion masses and mixings. To tackle the limitations of the SM, various extensions, including larger scalar and/or fermion sectors, as well as extended gauge groups with additional flavor symmetries, have been proposed in the literature [4-56]. Recent reviews on flavor symmetries are provided in refs. [57-62]. Another approach to describe the fermion mass and mixing pattern consists in postulating particular mass matrix textures (see refs. [63-93] for works which consider textures). In addition, the hierarchy of SM charged fermion masses can also be explained by considering the charged fermion Yukawa matrices as products of a few random matrices, which typically feature strong hierarchies in their eigenvalue spectrum, even though the individual entries are of order unity, as was recently observed in ref. [94].

Concerning models with an extended gauge symmetry, those based on the gauge symmetry $\mathrm{SU}(3)_{c} \times \mathrm{SU}(3)_{L} \times \mathrm{U}(1)_{X}$, also called 3-3-1 models, which introduce a family nonuniversal $\mathrm{U}(1)_{X}$ symmetry [95-106], can provide an explanation for the origin of the family structure of the fermions. These models have the following phenomenological advantages: (i) The three family structure in the fermion sector can be understood in the 3-3-1 models from the cancellation of chiral anomalies and asymptotic freedom in QCD. (ii) The fact that the third family is treated under a different representation can explain the large mass difference between the heaviest quark family and the two lighter ones. (iii) The 3-3-1 models allow for the quantization of electric charge [107, 108]. (iv) These models have several sources of CP violation $[109,110]$. (v) These models explain why the Weinberg mixing angle satisfies $\sin ^{2} \theta_{W}<\frac{1}{4}$. (vi) These models contain a natural Peccei-Quinn symmetry, which solves the strong-CP problem [111-114]. (vii) The 3-3-1 models with heavy sterile neutrinos include cold dark matter candidates as weakly interacting massive particles (WIMPs) [115-118]. A concise review of WIMPs in 3-3-1 Electroweak Gauge Models is provided in ref. [119].

In most versions of 3-3-1 models, one heavy triplet field with a Vacuum Expectation Value $(\mathrm{VEV})$ at a high energy scale breaks the symmetry $\mathrm{SU}(3)_{L} \times \mathrm{U}(1)_{X}$ into the SM electroweak group $\mathrm{SU}(2)_{L} \times \mathrm{U}(1)_{Y}$, thus generating masses for the non SM fermions and non SM gauge bosons, while other two lighter triplets with VEVs at the electroweak scale, trigger the Electroweak Symmetry Breaking [80] and provide the masses for the SM particles. To provide an explanation for the observed pattern of SM fermion masses and mixings, various 3-3-1 models with flavor symmetries [33-45, 120-123] and radiative seesaw mechanisms [80, 120, 124-133] have been proposed in the literature. However, some of them involve non renormalizable interactions [39, 40, 42, 43, 45], others are renormalizable but do not address the observed pattern of fermion masses and mixings due to the unexplained huge hierarchy among the Yukawa couplings [33, 35-38, 122, 123, 134] and others are only focused either in the quark mass hierarchy [34, 128, 130], or in the study of the neutrino sector [120, 124-127, 129, 132, 133, 135, 136], or only include the description of SM fermion mass hierarchy, without addressing the mixings in the fermion sector [131]. It is interesting 
to find an alternative explanation for the observed SM fermion mass and mixing pattern, in the framework of 3-3-1 models, by considering that it arises by a sequential loop suppression, so that the masses are generated according to: three level top quark mass, one loop level bottom, charm, tau and muon masses and two loop level masses for the light up, down and strange quarks as well as for the electron and neutrinos. This way of generating the SM fermion mass hierarchy was proposed for the first time in ref. [137]. However, the proposed model includes non-renormalizable Yukawa terms with a quite low cutoff scale. In this paper we propose the first renormalizable extension of the 3-3-1 model with the electric charge constructed from the $\mathrm{SU}(3)_{L}$ generators as $Q=T_{3}+\beta T_{8}+X I$ with $\beta=-\frac{1}{\sqrt{3}}$. The model explains the SM fermion mass and mixing pattern by a sequential loop suppression mechanism.

The paper is organized as follows. In section 2 we present the theoretical setup of the proposed model. In section 3 we discuss the quark masses and mixings within the model, while the discussion of the lepton masses and mixings is given in section 4 .

\section{The model}

The $\mathrm{SU}(3)_{C} \times S U(3)_{L} \times U(1)_{X}$ model (3-3-1 model) with $\beta=-\frac{1}{\sqrt{3}}$ and right-handed Majorana neutrinos in the $\mathrm{SU}(3)_{L}$ lepton triplet was proposed for the first time in [138]. However, the observed pattern of fermion masses and mixings was not addressed at that time due to the unexplained huge hierarchy among the Yukawa couplings [122, 129, 134]. Here we propose the first renormalizable extension of the 3-3-1 model with the parameter $\beta=-\frac{1}{\sqrt{3}}$, which includes a loop suppression mechanism to generate the observed pattern of the SM fermion masses and mixings. In our model only the top quark and the charged exotic fermions acquire tree level masses, whereas the remaining SM fermions get their masses via radiative corrections: 1 loop bottom, charm, tau and muon masses; 2-loop masses for the light up, down, strange quarks as well as for the electron. Light active neutrinos acquire their masses from a combination of linear and inverse seesaw mechanisms at two loop level, and the quark mixings arise from a combination of one and two loop level effects.

In order to realize this scenario we extend the $\mathrm{SU}(3)_{C} \times \mathrm{SU}(3)_{L} \times \mathrm{U}(1)_{X}$ group with an extra $Z_{4} \times Z_{2}$ discrete group, where the $Z_{4}$ symmetry is softly broken and the remaining $Z_{2}$ symmetry is broken both spontaneously and softly. We also introduce a global $\mathrm{U}(1)_{L_{g}}$ of the generalized lepton number $L_{g}$ [127], which is spontaneously broken down to a residual discrete $Z_{2}^{\left(L_{g}\right)}$ lepton number symmetry by a VEV of a gauge-singlet scalar $\xi^{0}$ to be introduced below. The corresponding massless Goldstone boson, Majoron, is phenomenologically harmless being a gauge-singlet. The full symmetry $\mathcal{G}$ of the model experiences a two-step spontaneous breaking, as follows:

$$
\begin{aligned}
\mathcal{G} & =\mathrm{SU}(3)_{C} \times \mathrm{SU}(3)_{L} \times \mathrm{U}(1)_{X} \times Z_{4} \times Z_{2} \times \mathrm{U}(1)_{L_{g}} \\
\stackrel{v_{\chi}, v_{\xi}}{\longrightarrow} & \mathrm{SU}(3)_{C} \times \mathrm{SU}(2)_{L} \times \mathrm{U}(1)_{Y} \times Z_{4} \times Z_{2}^{\left(L_{g}\right)} \\
\stackrel{v_{\eta}}{\longrightarrow} & \mathrm{SU}(3)_{C} \times \mathrm{U}(1)_{e m} \times Z_{4} \times Z_{2}^{\left(L_{g}\right)},
\end{aligned}
$$


where the different symmetry breaking scales satisfy the following hierarchy

$$
v_{\eta}=v=246 \mathrm{GeV} \ll v_{\chi} \sim v_{\xi} \sim \mathcal{O}(10) \mathrm{TeV},
$$

which corresponds in our model to the VEVs of the scalar fields to be introduced below. In the 3-3-1 model under consideration, the electric charge is defined as [96, 122, 138]:

$$
Q=T_{3}+\beta T_{8}+X I=T_{3}-\frac{1}{\sqrt{3}} T_{8}+X I
$$

where $T_{3}$ and $T_{8}$ are the $\mathrm{SU}(3)_{L}$ diagonal generators, $I$ is the $3 \times 3$ identity matrix and $X$ is the $\mathrm{U}(1)_{X}$ charge.

Different versions of the 3-3-1 models are determined by the choice of the $\beta$ parameter, which is related to the different possible fermion assignments. The most studied versions of 3-3-1 models have $\beta= \pm \frac{1}{\sqrt{3}}[95,138]$ and $\beta= \pm \sqrt{3}[97,99,139]$, and if we want to avoid exotic charges we are led to only two different models: $\beta= \pm \frac{1}{\sqrt{3}}$. Those having $\beta= \pm \frac{1}{\sqrt{3}}$ contain non SM fermions with non-exotic electric charges, i.e., equal to the electric charge of some SM fermions [140-142]. Those with $\beta= \pm \sqrt{3}$ have non SM fermions with large exotic electric charges and require a departure from the perturbative regime at a scale of several $\mathrm{TeV}$, in order to successfully account for the measured value of the weak mixing angle at low energies, as shown in detail in ref. [143]. Other versions of 3-3-1 models have $\beta=0, \pm \frac{2}{\sqrt{3}}$ and contain non SM particles with fractional electric charges [144]. For instance, 3-3-1 models with $\beta=0$ contains exotic quarks and exotic charged leptons with electric charges $\frac{1}{6}$ and $-\frac{1}{2}$, respectively [144]. Since electric charge conservation implies that the lightest exotic particles of the 3-3-1 models with $\beta=0, \pm 2 / \sqrt{3}$ should be stable, the phenomenological viability of such models requires a detailed analysis of the abundance of such stable exotic charged particles in cosmology.

For these reasons, 3-3-1 models with $\beta=-\frac{1}{\sqrt{3}}$ have advantages over those with $\beta=$ $0, \pm \frac{2}{\sqrt{3}}, \pm \sqrt{3}$. In addition, choosing $\beta=-\frac{1}{\sqrt{3}}$ implies that the third component of the weak lepton triplet is a neutral field $\nu_{R}^{C}$, which allows building the Dirac matrix with the usual field $\nu_{L}$ of the weak doublet. If one introduces a sterile neutrino $N_{R}$ in the model, the light neutrino masses can be generated via low scale seesaw mechanisms, which could be inverse or linear. The 3-3-1 models with $\beta=-\frac{1}{\sqrt{3}}$ can also provide an alternative framework to generate neutrino masses, where the neutrino spectrum includes the light active sub-eV scale neutrinos, as well as sterile neutrinos, which could be dark matter candidates, if they are light enough, or candidates for detection at the LHC, if their masses are at the $\mathrm{TeV}$ scale. Therefore, pair production of $\mathrm{TeV}$ scale sterile neutrinos via the Drell-Yan mechanism at the LHC could be a signal supporting models with extended gauge symmetries such as the 3-3-1 models. In addition, Drell-Yan heavy vector pair production processes at the LHC may help to distinguish the 3-3-1 models from other models with extended gauge symmetry. With respect to the quark spectrum, we assign each of the first two families of quarks to an $\mathrm{SU}(3)_{L}$ antitriplet $3^{*}$, whereas the third family is assigned to a $\mathrm{SU}(3)_{L}$ triplet 3 , as required by the $\mathrm{SU}(3)_{L}$ anomaly cancellation condition. Therefore, considering that there are 3 quark colors, we have six $3^{*}$ irreducible 
representations. In addition, there are six $\mathrm{SU}(3)_{L}$ triplets 3 of fermionic fields, considering the three lepton families. Thus, the $\mathrm{SU}(3)_{L}$ representations are vector like and anomaly free. The quantum numbers for the fermion families are assigned in such a way that the combination of the $\mathrm{U}(1)_{X}$ representations with other gauge sectors is anomaly free. As a consequence, one finds that the number of chiral fermion generations is an integer multiple of the number of colors, which provides an explanation for the existence of three generations of quarks and leptons in terms of the 3 colors. The $\mathrm{U}(1)_{X}$-charge assignments of the fermionic fields are obtained from eq. (2.3) and the requirement of reproducing the electric charges of the SM quarks and leptons. Then the $\mathrm{U}(1)_{X}$ charge of the first two families of quark antitriplets is $X_{Q_{n L}}=\frac{1}{6}+\frac{\beta}{2 \sqrt{3}}(n=1,2)$, whereas for the third family of quark triplet is $X_{Q_{3 L}}=\frac{1}{6}-\frac{\beta}{2 \sqrt{3}}$, and the corresponding $\mathrm{U}(1)_{X}$-charges of the right handed quarks are equal to their electric charges, given by $X_{u_{j R}, d_{j R}, J_{n R}}=\frac{2}{3},-\frac{1}{3}, \frac{1}{6}+\frac{\sqrt{3}}{2} \beta$, $(j=1,2,3$ and $n=1,2)$. The third generation non SM right handed quark $T_{R}$ has a $\mathrm{U}(1)_{X}$-charge given by $X_{T_{R}}=\frac{1}{6}-\frac{\sqrt{3}}{2} \beta$. The three left-handed lepton families are grouped into $\mathrm{SU}(3)_{L}$ triplets with $X_{L_{j L}}=-\frac{1}{2}-\frac{\beta}{2 \sqrt{3}}(j=1,2,3)$, while the right-handed leptons are assigned as $\mathrm{SU}(3)_{L}$ singlets with $\mathrm{U}(1)_{X}$-charges equal to their electric charges, given by $X_{e_{i R}, \tilde{e}_{i R}}=-1,-\frac{1}{2}-\frac{\sqrt{3}}{2} \beta$, where $\tilde{e}_{i R}$ are the right handed exotic leptons. These exotic fermions reside in vector-like representations of the SM gauge group and are singlets under the $\mathrm{SU}(2)_{L}$. Since we are considering a 3-3-1 model with $\beta=-\frac{1}{\sqrt{3}}$, the cancellation of chiral anomalies implies that quarks are unified in the following $\mathrm{SU}(3)_{C} \times \mathrm{SU}(3)_{L} \times \mathrm{U}(1)_{X}$ left- and right-handed representations [96, 101, 145, 146]:

$$
\begin{aligned}
& Q_{n L}=\left(\begin{array}{c}
D_{n} \\
-U_{n} \\
J_{n}
\end{array}\right)_{L} \sim\left(3,3^{*}, 0\right), \quad Q_{3 L}=\left(\begin{array}{c}
U_{3} \\
D_{3} \\
T
\end{array}\right)_{L} \sim\left(3,3, \frac{1}{3}\right), \quad n=1,2, \\
& D_{i R} \sim\left(3,1,-\frac{1}{3}\right), \quad U_{i R} \sim\left(3,1, \frac{2}{3}\right), \quad i=1,2,3, \\
& J_{n R} \sim\left(3,1,-\frac{1}{3}\right), \quad T_{R} \sim\left(3,1, \frac{2}{3}\right),
\end{aligned}
$$

where $U_{i L}$ and $D_{i L}(i=1,2,3)$ are the left handed up and down type quarks fields in the flavor basis, respectively. The right handed SM quarks, i.e., $U_{i R}$ and $D_{i R}(i=1,2,3)$ and right handed exotic quarks, i.e., $T_{R}$ and $J_{n R}(n=1,2)$ are assigned to be $\mathrm{SU}(3)_{L}$ singlets with $\mathrm{U}(1)_{X}$ quantum numbers equal to their electric charges.

Furthermore, the requirement of chiral anomaly cancellation constrains the leptons to the following $\mathrm{SU}(3)_{C} \times \mathrm{SU}(3)_{L} \times \mathrm{U}(1)_{X}$ left- and right-handed representations $[96,101,145]$ :

$$
L_{i L}=\left(\begin{array}{c}
\nu_{i} \\
e_{i} \\
\nu_{i}^{c}
\end{array}\right)_{L} \sim\left(1,3,-\frac{1}{3}\right), \quad e_{i R} \sim(1,1,-1), \quad i=1,2,3,
$$

where $\nu_{i L}, \nu^{c} \equiv \nu_{R}^{c}$ and $e_{i L}\left(e_{L}, \mu_{L}, \tau_{L}\right)$ are the neutral and charged lepton families, respectively. Let us note that we assign the right-handed leptons to $\mathrm{SU}(3)_{L}$ singlets, which implies that their $\mathrm{U}(1)_{X}$ quantum numbers correspond to their electric charges. 


\begin{tabular}{|c|c|c|c|c|c|c|c|c|c|c|c|c|c|c|c|c|c|c|}
\hline & $Q_{1 L}$ & $Q_{2 L}$ & $Q_{3 L}$ & $U_{1 R}$ & $U_{2 R}$ & $U_{3 R}$ & $T_{R}$ & $D_{1 R}$ & $D_{2 R}$ & $D_{3 R}$ & $J_{1 R}$ & $J_{2 R}$ & $\widetilde{T}_{1 L}$ & $\widetilde{T}_{1 R}$ & $\widetilde{T}_{2 L}$ & $\widetilde{T}_{2 R}$ & $B_{L}$ & $B_{R}$ \\
\hline$L_{g}$ & $\frac{2}{3}$ & $\frac{2}{3}$ & $-\frac{2}{3}$ & 0 & 0 & 0 & -2 & 0 & 0 & 0 & 2 & 2 & 0 & 0 & 0 & 0 & 0 & 0 \\
\hline$Z_{4}$ & -1 & -1 & 1 & 1 & $-i$ & 1 & 1 & 1 & 1 & 1 & -1 & -1 & $i$ & 1 & $i$ & 1 & -1 & -1 \\
\hline$Z_{2}$ & 1 & 1 & 1 & 1 & 1 & -1 & -1 & 1 & 1 & 1 & -1 & -1 & 1 & 1 & 1 & 1 & 1 & 1 \\
\hline
\end{tabular}

Table 1. Quark assignments under $Z_{4} \times Z_{2}$ and the values of generalized Lepton Number $L_{g}$.

\begin{tabular}{|c|c|c|c|c|c|c|c|c|c|c|c|c|c|c|c|c|}
\hline & $L_{1 L}$ & $L_{2 L}$ & $L_{3 L}$ & $e_{1 R}$ & $e_{2 R}$ & $e_{3 R}$ & $E_{1 L}$ & $E_{2 L}$ & $E_{3 L}$ & $E_{1 R}$ & $E_{2 R}$ & $E_{3 R}$ & $N_{1 R}$ & $N_{2 R}$ & $N_{3 R}$ & $\Psi_{R}$ \\
\hline$L_{g}$ & $\frac{1}{3}$ & $\frac{1}{3}$ & $\frac{1}{3}$ & 1 & 1 & 1 & 1 & 1 & 1 & 1 & 1 & 1 & -1 & -1 & -1 & 1 \\
\hline$Z_{4}$ & $i$ & $i$ & $i$ & $-i$ & $-i$ & $-i$ & 1 & $i$ & $i$ & $-i$ & $-i$ & $-i$ & $i$ & $i$ & $i$ & 1 \\
\hline$Z_{2}$ & -1 & 1 & 1 & -1 & 1 & 1 & -1 & 1 & 1 & -1 & 1 & 1 & -1 & -1 & -1 & -1 \\
\hline
\end{tabular}

Table 2. Lepton assignments under $Z_{4} \times Z_{2}$ and the values of generalized Lepton Number $L_{g}$.

To implement the radiative seesaw mechanisms that generate the observed hierarchy of the SM charged fermion masses and mixing angles by a sequential loop suppression and the light active neutrino masses from a combination of linear and inverse seesaw mechanisms at two loop level, we extend both the fermion and the scalar sectors of the 3-3-1 models with $\beta=-\frac{1}{\sqrt{3}}$ previously considered in the literature. We introduce $\mathrm{SU}(3)_{L}$ singlet exotic up type quarks $\widetilde{T}_{L, R}$, down type quarks $B_{L, R}$ and charged leptons $E_{L, R}$ as well as four gauge group eq. (2.1) singlet leptons $N_{R}, \Psi_{R}$. Their complete $\mathrm{SU}(3)_{C} \times \mathrm{SU}(3)_{L} \times \mathrm{U}(1)_{X}$ assignments are:

$$
\begin{aligned}
& \widetilde{T}_{1 L} \sim(3,1,2 / 3), \quad \widetilde{T}_{1 R} \sim(3,1,2 / 3), \quad \widetilde{T}_{2 L} \sim(3,1,2 / 3), \quad \widetilde{T}_{2 R} \sim(3,1,2 / 3), \\
& B_{L} \sim(3,1,-1 / 3), \quad B_{R} \sim(3,1,-1 / 3), \\
& E_{1 L} \sim(1,1,-1), \quad E_{2 L} \sim(1,1,-1), \quad E_{3 L} \sim(1,1,-1), \\
& E_{1 R} \sim(1,1,-1), \quad E_{2 R} \sim(1,1,-1), \quad E_{3 R} \sim(1,1,-1), \\
& N_{1 R} \sim(1,1,0), \quad N_{2 R} \sim(1,1,0), \quad N_{3 R} \sim(1,1,0), \quad \Psi_{R} \sim(1,1,0) .
\end{aligned}
$$

The $\mathrm{U}(1)_{L_{g}} \times Z_{4} \times Z_{2}$ assignments for all the fermions of the model are shown in tables 1,2 .

Compared to the simplified versions of 3-3-1 models with the scalar sector composed only of three $\mathrm{SU}(3)_{L}$ scalar triplets $-\chi, \eta$ and $\rho$ - we introduce seven $\mathrm{SU}(3)_{L}$ singlets $\varphi_{1}^{0}, \varphi_{2}^{0}, \xi^{0}, \phi_{1}^{+}, \phi_{2}^{+}, \phi_{3}^{+}$and $\phi_{4}^{+}$. All these scalars are assigned in our model to the following representations of $\mathrm{SU}(3)_{C} \times \mathrm{SU}(3)_{L} \times \mathrm{U}(1)_{X}$ :

$$
\begin{aligned}
& \chi=\left(\begin{array}{c}
\chi_{1}^{0} \\
\chi_{2}^{-} \\
\frac{1}{\sqrt{2}}\left(v_{\chi}+\xi_{\chi} \pm i \zeta_{\chi}\right)
\end{array}\right) \sim\left(1,3,-\frac{1}{3}\right), \quad \rho=\left(\begin{array}{c}
\rho_{1}^{+} \\
\frac{1}{\sqrt{2}}\left(\xi_{\rho} \pm i \zeta_{\rho}\right) \\
\rho_{3}^{+}
\end{array}\right) \sim\left(1,3, \frac{2}{3}\right), \\
& \eta=\left(\begin{array}{c}
\frac{1}{\sqrt{2}}\left(v_{\eta}+\xi_{\eta} \pm i \zeta_{\eta}\right) \\
\eta_{2}^{-} \\
\eta_{3}^{0}
\end{array}\right) \sim\left(1,3,-\frac{1}{3}\right), \quad \varphi_{1}^{0} \sim(1,1,0), \quad \varphi_{2}^{0} \sim(1,1,0), \\
& \phi_{1}^{+} \sim(1,1,1), \quad \phi_{2}^{+} \sim(1,1,1), \quad \phi_{3}^{+} \sim(1,1,1), \quad \phi_{4}^{+} \sim(1,1,1), \quad \xi^{0} \sim(1,1,0),
\end{aligned}
$$

Their $\mathrm{U}(1)_{L_{g}} \times Z_{4} \times Z_{2}$ assignments are shown in table 3 . 


\begin{tabular}{|c|c|c|c|c|c|c|c|c|c|c|}
\hline & $\chi$ & $\eta$ & $\rho$ & $\varphi_{1}^{0}$ & $\varphi_{2}^{0}$ & $\phi_{1}^{+}$ & $\phi_{2}^{+}$ & $\phi_{3}^{+}$ & $\phi_{4}^{+}$ & $\xi^{0}$ \\
\hline$L_{g}$ & $\frac{4}{3}$ & $-\frac{2}{3}$ & $-\frac{2}{3}$ & 0 & 0 & 0 & -2 & -2 & -2 & -2 \\
\hline$Z_{4}$ & 1 & 1 & -1 & -1 & $i$ & $i$ & -1 & -1 & 1 & 1 \\
\hline$Z_{2}$ & -1 & -1 & 1 & 1 & 1 & 1 & 1 & -1 & -1 & 1 \\
\hline
\end{tabular}

Table 3. Scalar assignments under $Z_{4} \times Z_{2}$ and the values of generalized Lepton Number $L_{g}$.

The spontaneous symmetry breaking (2.1) in our model is triggered by the VEVs $(2.2)$ of the scalar fields $\chi, \eta$ and $\xi^{0}$, neutral under the $Z_{4}$ discrete symmetry. As seen from (2.1), the first stage of the breaking is done by a TeV scale VEV $v_{\chi}$ of an $\mathrm{SU}(3)_{L}$ triplet $\chi$ handing masses to the non-SM fermions and gauge bosons as well as by the TeV scale VEV $v_{\xi}$ of the gauge-singlet scalar $\xi^{0}$, which spontaneously breaks the generalized lepton number symmetry $\mathrm{U}(1)_{L_{g}}$. The corresponding Majoron is a gauge-singlet and, therefore, unobservable. Note that Lepton Number (LN) is broken together with Generalized Lepton Number (GLN) by the VEV of $\xi^{0}$, which has both LN and GLN equal to -2 . Since the gauge singlet scalar $\xi^{0}$ breaks $\mathrm{U}(1)_{L_{g}}$ in a way that respects the condition $\left|\Delta L_{g}\right|=|\Delta L|=2$, there survives a residual discrete $Z_{2}^{\left(L_{g}\right)}$ lepton number symmetry under which the leptons are charged and the other particles are neutral. This means that in any reaction leptons can appear only in pair, thus, forbidding proton decay. The TeV scale VEVs $v_{\chi}$ of the $\mathrm{SU}(3)_{L}$ triplet $\chi$ also breaks $Z_{2}$ symmetry. Another $\mathrm{SU}(3)_{L}$ triplet $\eta$ with a Fermi scale VEV $v_{\eta}$ is responsible for the electroweak symmetry breaking and the masses of the SM fermions and $W, Z$-bosons.

Let us explain the VEV pattern of the $\mathrm{SU}(3)_{L}$ scalar triplets $\chi$ and $\eta$. Since the $\chi$ triggers the $\mathrm{SU}(3)_{L} \times \mathrm{U}(1)_{X} \rightarrow \mathrm{SU}(2)_{L} \times \mathrm{U}(1)_{Y}$ breaking, the following conditions have to be fulfilled:

$$
T_{1}\langle\chi\rangle=T_{2}\langle\chi\rangle=T_{3}\langle\chi\rangle=\left(\beta T_{8}+X I\right)\langle\chi\rangle=0 .
$$

whereas the remaining generators do not leave the vacuum $\langle\chi\rangle$ invariant. From the first three conditions for $\langle\chi\rangle$ given in eq. (2.9), it follows that:

$$
\langle\chi\rangle=\left(\begin{array}{c}
0 \\
0 \\
\frac{v_{\chi}}{\sqrt{2}}
\end{array}\right)
$$

The last condition in eq. (2.9) for $\langle\chi\rangle$, i.e, $\left(\beta T_{8}+X I\right)\langle\chi\rangle=0$, yields the following relation between the $U(1)_{X}$ charge of the $\mathrm{SU}(3)_{L}$ scalar triplet $\chi$ and the $\beta$ parameter:

$$
X_{\chi}=\frac{\beta}{\sqrt{3}},
$$

which for $\beta=-\frac{1}{\sqrt{3}}$, results in $X_{\chi}=-\frac{1}{3}$, as indicated by eq. (2.8). 
The electroweak symmetry breaking $\mathrm{SU}(2)_{L} \times \mathrm{U}(1)_{Y} \rightarrow \mathrm{U}(1)_{E M}$ in our model is realized by the $\mathrm{VEV} v_{\eta}$ of the $\mathrm{SU}(3)_{L}$ scalar triplet $\eta$. Requiring that all the $S U(3)_{L}$ generators are broken, with the exception of the electric charge generator $Q$, we arrive at the following VEV pattern

$$
\langle\eta\rangle=\left(\begin{array}{c}
\frac{v_{\eta}}{\sqrt{2}} \\
0 \\
0
\end{array}\right) .
$$

From the requirement of the $\mathrm{U}(1)_{E M}$ invariance we have

$$
Q\langle\eta\rangle=\left(T_{3}+\beta T_{8}+X I\right)\langle\eta\rangle=0,
$$

thus producing the following relation for the $\mathrm{U}(1)_{X}$ charge $X_{\eta}$ of the $\eta$ field:

$$
X_{\eta}=-\frac{1}{2}-\frac{\beta}{2 \sqrt{3}}
$$

which for $\beta=-\frac{1}{\sqrt{3}}$ results in $X_{\eta}=-\frac{1}{3}$ as indicated by eq. (2.8).

Note that, the difference between the $\eta$ and $\chi$ Higgs triplets can be explained using the generalized lepton number $L_{g}$, discussed in appendix A. Its values for the fields of the model are specified in tables $1-3$.

The choice of the VEV structure in (2.10) and (2.12) shows that only the neutral Higgs field without lepton number is allowed to have the VEV. In addition, the patterns of the $\mathrm{SU}(3)_{L}$ scalar triplets $\chi$ and $\eta$ shown in eq. (2.10) and (2.12) are consistent with a global minimum of the scalar potential of our model for all the region of parameter space. We adopt $X_{\rho}=2 / 3$ for another $\mathrm{SU}(3)_{L}$ scalar triplet $\rho$ in eq. (2.8) from the simplified versions of the 3-3-1 model $[101,102,138]$, where both $\eta$ and $\rho$ scalars participate in the electroweak symmetry breaking. The extra $\mathrm{SU}(3)_{L}$ scalar triplet $\rho$ is introduced in simplified versions of the 3-3-1 models to give masses to charged leptons, as well as to the bottom, up and charm quarks. In our model the $\mathrm{SU}(3)_{L}$ scalar triplet $\rho$ is crucial to give one loop level masses for the bottom and charm quarks, to the tau and muon leptons as well as two loop level masses for the up, down and strange quarks as well to the electron, as shown in figures 1,2 . The $\mathrm{SU}(3)_{L}$ scalar triplet $\rho$ also contributes to some entries of the neutrino mass matrix as indicated in figure 3. On the other hand, the conditions similar to (2.12), (2.13) are applied to $\langle\rho\rangle$ as well and lead to $X_{\rho}=2 / 3$. In our model we have $\langle\rho\rangle=0$ due to the $Z_{4}$ conservation (2.1), and the above symmetry breaking conditions do not restrict $X_{\rho}$. We choose $X_{\rho}=2 / 3$ in order to maintain resemblance with the previous versions of the 3-3-1 model. Another motivation for the choice $X_{\rho}=2 / 3$ is the $\mathrm{U}(1)_{X}$ invariance of the $\mathrm{SU}(3)_{L}$ invariant trilinear scalar interaction $\chi \eta \rho$. Let us note that our choice $\beta=-\frac{1}{\sqrt{3}}$ yields $X_{\eta}=X_{\chi}=-\frac{1}{3}$, which in turn leads to $X_{\rho}=2 / 3$. 
With the above particle content, the relevant quark and lepton Yukawa terms invariant under the symmetry group (2.1) of our model take the form:

$$
\begin{aligned}
-L_{g Y}^{(q)}= & h_{\chi}^{(T)} \bar{Q}_{3 L} \chi T_{R}+h_{\eta}^{(U)} \bar{Q}_{3 L} \eta U_{3 R} \\
& +\sum_{n=1}^{2} \sum_{m=1}^{2} h_{\rho n m}^{(\widetilde{T})} \bar{Q}_{n L} \rho^{*} \widetilde{T}_{m R}+\sum_{n=1}^{2} h_{\varphi_{1}^{0} n 2}^{(U)} \overline{\widetilde{T}}_{n L} \varphi_{1}^{0} U_{2 R}+\sum_{n=1}^{2} h_{\varphi_{2}^{0} n 1}^{(U)} \overline{\widetilde{T}}_{n L} \varphi_{2}^{0} U_{1 R} \\
& +\sum_{n=1}^{2} \sum_{m=1}^{2} h_{\chi n m}^{(J)} \bar{Q}_{n L} \chi^{*} J_{m R}+h_{\rho}^{(B)} \bar{Q}_{3 L} \rho B_{R}+\sum_{j=1}^{3} h_{\varphi_{1}^{0} j}^{(D)} \bar{B}_{L} \varphi_{1}^{0} D_{j R} \\
& +\sum_{n=1}^{2} \sum_{j=1}^{3} h_{\phi_{1}^{+} n j}^{(D)} \overline{\widetilde{T}}_{n L} \phi_{1}^{+} D_{j R}+\sum_{n=1}^{2} \sum_{m=1}^{2} h_{\varphi_{2}^{0} n m}^{(\widetilde{T})} \overline{\widetilde{T}}_{n L} \varphi_{2}^{0} \widetilde{T}_{m R}+m_{B} \bar{B}_{L} B_{R}+h . c, \\
-L_{g Y}^{(l)}= & h_{\rho}^{(E)} \bar{L}_{1 L} \rho E_{1 R}+h_{\varphi_{2}^{0}}^{(E)} \bar{E}_{1 L} \varphi_{2}^{0} E_{1 R}+h_{\varphi_{2}^{0}}^{(e)} \bar{E}_{1 L} \varphi_{2}^{0} e_{1 R}+\sum_{n=2}^{3} \sum_{m=2}^{3} h_{\rho n m}^{(E)} \bar{L}_{n L} \rho E_{m R} \\
& +h_{\rho}^{(e)} \bar{L}_{1 L} \rho e_{1 R}+\sum_{n=2}^{3} \sum_{m=2}^{3} h_{\rho n m}^{(e)} \bar{L}_{n L} \rho e_{m R}+\sum_{n=2}^{3} \sum_{m=2}^{3} h_{\varphi_{1}^{0} n m}^{(E)} \bar{E}_{n L} \varphi_{1}^{0} E_{m R} \\
& +\sum_{n=2}^{3} \sum_{m=2}^{3} h_{\varphi_{1}^{0} n m}^{(e)} \bar{E}_{n L} \varphi_{1}^{0} e_{m R}+\sum_{n=2}^{3} \sum_{j=1}^{3} h_{\chi n j}^{(L)} \bar{L}_{n L} \chi N_{j R} \\
& +\sum_{j=1}^{3} \sum_{n=2}^{3} h_{\phi_{4}^{-} n j}^{(e)} \bar{E}_{n L} \phi_{4}^{-} N_{j R}+\sum_{j=1}^{3} h_{\varphi_{2}^{0}}^{(N)} \bar{\Psi}_{R}^{c}\left(\varphi_{2}^{0}\right)^{*} N_{j R}+y_{\Psi} \bar{\Psi}_{R}^{c} \Psi_{R} \xi^{0} \\
& +h_{\rho 11}^{(L)} \varepsilon_{a b c} \bar{L}_{1 L}^{a}\left(L_{1 L}^{C}\right)^{b}\left(\rho^{*}\right)^{c}+\sum_{n=2}^{3} \sum_{m=2}^{3} h_{\rho n m}^{(L)} \varepsilon_{a b c} \bar{L}_{n L}^{a}\left(L_{m L}^{C}\right)^{b}\left(\rho^{*}\right)^{c}+h . c .
\end{aligned}
$$

where the dimensionless parameters in eqs. (2.16) and (2.16) are $\mathcal{O}(1)$ dimensionless couplings. From the quark Yukawa terms it follows that the top quark mass mainly arises from the interaction with the $\mathrm{SU}(3)_{L}$ scalar triplet $\eta$, which breaks the $S U(2)_{L} \times U(1)_{Y}$ gauge group. Consequently, the dominant contribution to the SM-like $126 \mathrm{GeV}$ Higgs boson arises mainly from the $\mathrm{CP}$ even neutral component $\xi_{\eta}$ of the $\mathrm{SU}(3)_{L}$ scalar triplet $\eta$. The terms of the scalar potential relevant for the implementation of the radiative seesaw mechanisms that generate the observed hierarchy of the SM charged fermion masses and mixing angles by a sequential loop suppression are:

$$
\begin{aligned}
V \supset & \lambda_{1} \eta \chi \rho \varphi_{1}^{0}+\lambda_{2} \eta \chi \rho\left(\varphi_{1}^{0}\right)^{*}+\lambda_{3} \phi_{3}^{-} \rho \eta^{\dagger} \xi^{0}+\lambda_{4} \phi_{1}^{-} \phi_{2}^{+}\left(\varphi_{2}^{0}\right)^{*}\left(\xi^{0}\right)^{*} \\
& +w_{1}\left(\varphi_{2}^{0}\right)^{2} \varphi_{1}^{0}+w_{2} \phi_{3}^{-} \rho \chi^{\dagger}+\text { h.c. }
\end{aligned}
$$

After the spontaneous breaking of the electroweak symmetry, the above-given Yukawa interactions generate the observed hierarchy of SM fermion masses and mixing angles by a sequential loop suppression, provided that one introduces the $Z_{4} \times Z_{2}$ soft breaking mass 
terms for the electroweak singlet fermions:

$$
\begin{aligned}
L_{\text {gsoft }}^{F}= & \sum_{n=1}^{2} \sum_{m=1}^{2}\left(m_{\widetilde{T}}\right)_{n m} \overline{\widetilde{T}}_{n L} \widetilde{T}_{m R}+m_{E_{1}} \bar{E}_{1 L} E_{1 R}+\sum_{n=2}^{3} \sum_{m=2}^{3}\left(m_{E}\right)_{n m} \bar{E}_{n L} E_{m R} \\
& +\sum_{n=2}^{3}\left(m_{E}\right)_{n 1} \bar{E}_{n L} E_{1 R}+\text { h.c. }
\end{aligned}
$$

as well as soft $Z_{4} \times Z_{2}$ breaking in the electroweak singlet scalar sector:

$$
L_{\text {gsoft }}^{\text {scalars }}=\mu_{1}^{2}\left(\varphi_{2}^{0}\right)^{2}+\mu_{2}^{2} \phi_{2}^{-} \phi_{3}^{+}+\mu_{3}^{2} \phi_{4}^{-} \phi_{3}^{+}+\text {h.c. } .
$$

Let us note that in the simplified version of the $3-3-1$ model with $\beta=-\frac{1}{\sqrt{3}}$, whose scalar sector contains three $\mathrm{SU}(3)_{L}$ scalar triplets, the flavor constraints can be fulfilled by considering the scale of breaking of the $\mathrm{SU}(3)_{L} \times \mathrm{U}(1)_{X}$ gauge symmetry much larger than the electroweak symmetry breaking scale $v=246 \mathrm{GeV}$, which corresponds to the alignment limit of the mass matrix for the CP-even Higgs bosons [147]. Our model has a more extended scalar sector since it is composed of three $\mathrm{SU}(3)_{L}$ scalar triplets (from which one is inert $\mathrm{SU}(3)_{L}$ triplet) and six $\mathrm{SU}(3)_{L}$ scalar singlets. Consequently, following ref. [147], we expect that the FCNC effects as well as the constraints arising from $K^{0}-\bar{K}^{0}, B^{0}-\bar{B}^{0}$ and $D^{0}-\overline{D^{0}}$ mixings will be fulfilled in our model, by considering the scale of breaking of the $\mathrm{SU}(3)_{L} \times \mathrm{U}(1)_{X}$ gauge symmetry much larger than the scale of breaking of the electroweak symmetry. The scalar sector of our model is not predictive as its corresponding scalar potential has many free uncorrelated parameters that can be adjusted to get the required pattern of scalar masses. Therefore, the loop effects of the heavy scalars contributing to certain observables can be suppressed by the appropriate choice of the free parameters in the scalar potential. Fortunately, all these adjustments do not affect the charged fermion and neutrino sector, which is completely controlled by the fermion-Higgs Yukawa couplings.

Despite the fact that the scalar and fermion sectors of our model are considerably larger than the corresponding to the simplified version of the 3-3-1 model with $\beta=-\frac{1}{\sqrt{3}}$, and the fields assignments under the discrete group $Z_{4} \times Z_{2}$ look rather sophisticated, each introduced element plays its own role in the implementation of the radiative seesaw mechanisms that allow us to explain the SM fermion mass hierarchy by a sequential loop suppression. In what follows we provide a justification and summary of our above presented model setup:

1. The spontaneously and softly broken $Z_{2}$ symmetry is crucial to generate a two loop level electron mass as it distinguishes the first generation left leptonic triplet, i.e., $L_{1 L}$, neutral under $Z_{2}$ from the second and third generation ones i.e., $L_{2 L}$ and $L_{3 L}$ which are $Z_{2}$ even. This symmetry also separates the SM right handed charged leptonic field, i.e, $e_{1 R}$, which is $Z_{2}$ odd from the remaining $\mathrm{SM}$ right handed charged leptonic fields, i.e, $e_{2 R}$ and $e_{3 R}$, neutral under the $Z_{2}$ symmetry. This results in one loop level tau and muon lepton masses and a two loop level mass for the electron.

2. The softly broken $Z_{4}$ symmetry separates the third generation left handed quark fields from the first and second generation ones, giving rise to a tree level top and 
exotic quark masses and to radiatively generated masses for the remaining quarks. Besides that, the $Z_{4}$ symmetry differentiates the second generation right handed SM up quark fields, i.e., $U_{2 R}$, charged under this symmetry, from the first generation SM one, i.e., $U_{1 R}$, which is $Z_{4}$ neutral, thus giving rise to a one loop level charm quark mass and two loop level up quark mass.

3. The scalar sector of our model is composed of three $\mathrm{SU}(3)_{L}$ scalar triplets, i.e., $\chi$, $\eta$ and $\rho$, seven $\mathrm{SU}(3)_{L}$ scalar singlets, from which three are electrically neutral, i.e., $\varphi_{1}^{0}, \varphi_{2}^{0}$ and $\xi^{0}$ and four electrically charged, i.e., $\phi_{1}^{+}, \phi_{2}^{+}, \phi_{3}^{+}$and $\phi_{4}^{+}$. The inclusion of the spontaneously and softly broken $Z_{2}$ symmetry requires the introduction of a $\mathrm{SU}(3)_{L}$ scalar singlet $\phi_{3}^{+}$, which is odd under this symmetry. The presence of the $\mathrm{SU}(3)_{L}$ scalar singlet $\phi_{3}^{+}$, is needed in order to build the $Z_{2}$ invariant trilinear scalar interactions required to generate two loop level down and strange quark masses, as shown in figure 1. Besides that, in order to implement a two loop level radiative seesaw mechanism for the generation of the up, down and strange quark masses as well as the electron mass, the $Z_{4}$ charged $\mathrm{SU}(3)_{L}$ scalar singlets $\varphi_{1}^{0}, \varphi_{2}^{0}, \phi_{1}^{+}, \phi_{2}^{+}$(which do not acquire a vacuum expectation value) are also required in the scalar sector. The $Z_{4}$ charged $\mathrm{SU}(3)_{L}$ scalar singlet $\varphi_{1}^{0}$ is also needed for the implementation of the one loop level radiative seesaw mechanism that generates the charm, the bottom quark masses as well as the tau and muon lepton masses, as shown in figure 2. The $Z_{4}$ charged $\mathrm{SU}(3)_{L}$ scalar singlets $\varphi_{2}^{0}$ and $\phi_{3}^{+}$as well as the $\mathrm{SU}(3)_{L}$ scalar singlet $\phi_{4}^{+}$, neutral under $Z_{4}$ are also crucial for the implementation of two loop level linear and inverse seesaw mechanisms that give rise to the light active neutrino masses. The $\mathrm{SU}(3)_{L}$ scalar singlet $\xi^{0}$ is introduced to spontaneously break the $\mathrm{U}(1)_{L_{g}}$ generalized lepton number symmetry and thus giving rise to a tree-level mass for the right handed Majorana neutrino $\Psi_{R}$. Lets us note that $\xi^{0}$ is the only electrically neutral $\mathrm{SU}(3)_{L}$ scalar singlet that has a non-vanishing generalized Lepton Number $L_{g}$. It is crucial for generating two loop-level masses for the down and strange quarks. This is due to the fact that the electrically charged $\mathrm{SU}(3)_{L}$ scalar singlets $\phi_{2}^{+}$and $\phi_{3}^{+}$appearing in the two loop level diagrams that give rise to the down and strange quark masses carry non-vanishing generalized Lepton Numbers thus implying that the quartic scalar interaction $\lambda_{3} \phi_{3}^{-} \rho \eta^{\dagger} \xi^{0}$ is crucial to generate the masses for the down and strange quarks, as shown in figure 1 . Note that we assign non-vanishing generalized Lepton Numbers for $\phi_{2}^{+}$and $\phi_{3}^{+}$because $\phi_{3}^{+}$mix with $\phi_{4}^{+}$as well as with $\phi_{2}^{+}$via the soft breaking mass terms of eq. (2.19) and $\phi_{4}^{+}$carry a non-vanishing generalized Lepton Number as required from the invariance of the lepton Yukawa interaction $\bar{E}_{n L} \phi_{4}^{-} N_{j R}$ under the $\mathrm{U}(1)_{L_{g}}$ symmetry.

4. The fermion sector of the 3-3-1 model, with right-handed neutrinos $\nu_{R}^{c}$ in the $\mathrm{SU}(3)_{L}$ lepton triplet, is extended by introducing two $\mathrm{SU}(3)_{L}$ singlet exotic up type quarks, i.e. $\widetilde{T}_{1}$ and $\widetilde{T}_{2}$, a SU $(3)_{L}$ singlet exotic down type quark, i.e., $B$, three $\mathrm{SU}(3)_{L}$ singlet exotic charged leptons, i.e., $E_{j}(j=1,2,3)$ and four right handed Majorana neutrinos $N_{j R}(j=1,2,3), \Psi_{R}$. The $\mathrm{SU}(3)_{L}$ singlet exotic down type quarks, i.e. $B$, is crucial for the implementation of the one loop level radiative seesaw mechanism that generate 
the bottom quark mass. The $\mathrm{SU}(3)_{L}$ singlet exotic up type quarks, i.e., $\widetilde{T}_{1}$ and $\widetilde{T}_{2}$, are needed to generate a one loop level charm quark mass as well as two loop level down and strange quark masses. The three $\mathrm{SU}(3)_{L}$ singlet exotic charged leptons, i.e., $E_{j}(j=1,2,3)$, are required in order to provide the radiative seesaw mechanisms that generate one loop level tau and muon masses and two loop level electron mass. The four right handed Majorana neutrinos, i.e., $N_{j R}(j=1,2,3), \Psi_{R}$, are crucial for the implementation of the two loop level linear and inverse seesaw mechanisms that give rise to the light active neutrino masses. It is worth mentioning that out of these four right handed Majorana neutrinos, only $\Psi_{R}$ acquires a tree level mass, whereas the three remaining right handed Majorana neutrinos, i.e., $N_{j R}(j=1,2,3)$, get their masses via a one loop level radiative seesaw mechanism mediated by $\Psi_{R}$ and $\varphi_{2}^{0}$, as shown in figure 3.

In what follows we briefly comment on some phenomenological aspects of our model concerning LHC signals of non-SM fermions. From the quark Yukawa interactions it follows that the heavy exotic $\mathrm{SU}(3)_{L}$ singlet down (up) type quark(s), i.e., $B\left(\widetilde{T}_{n}(n=1,2)\right)$ will decay predominantly into a SM down (up) type quark and the $\operatorname{Re} \varphi_{1}^{0}$ or $\operatorname{Im} \varphi_{1}^{0}$ neutral scalar, which is identified as missing energy, due to the preserved $Z_{4}$ symmetry. Furthermore, from the lepton Yukawa interactions it follows that the heavy $\mathrm{SU}(3)_{L}$ singlet exotic charged leptons, i.e. $E_{j}(j=1,2,3)$, will have a dominant decay mode into a SM charged lepton and a neutral $\mathrm{CP}$ even $\xi_{\rho}$ or $\mathrm{CP}$ odd $\zeta_{\rho}$ scalar state, which can also be identified as missing energy, due to the preserved $Z_{4}$ symmetry. The exotic $\mathrm{SU}(3)_{L}$ singlet up type quarks, i.e. $\widetilde{T}_{1}$ and $\widetilde{T}_{2}$ and down type quark, i.e., $B$, are produced in pairs at the LHC via gluon fusion and the Drell-Yan mechanism, and the charged exotic leptons $E_{j}(j=1,2,3)$ are also produced in pairs but only via the Drell-Yan mechanism. Thus, observing an excess of events with respect to the SM background in the dijet and opposite sign dileptons final states at the LHC, can be a signal in support of this model. With respect to the exotic $T$, $J^{1}$ or $J^{2}$ quarks, they mainly decay into a top quark and either neutral or charged scalar. The precise signature of the decays of the exotic quarks depends on details of the spectrum and other parameters of the model. The present lower limits on the $Z^{\prime}$ gauge boson mass in 3-3-1 models arising from LHC searches, reach around $2.5 \mathrm{TeV}$ [148]. These bounds can be translated into limits of about $6.3 \mathrm{TeV}$ on the $\mathrm{SU}(3)_{C} \times S U(3)_{L} \times U(1)_{X}$ gauge symmetry breaking scale $v_{\chi}$. Furthermore, electroweak data from the decays $B_{s, d} \rightarrow \mu^{+} \mu^{-}$ and $B_{d} \rightarrow K^{*}(K) \mu^{+} \mu^{-}$set lower bounds on the $Z^{\prime}$ gauge boson mass ranging from $1 \mathrm{TeV}$ up to $3 \mathrm{TeV}[146,149-152]$. The exotic quarks can be pair produced at the LHC via DrellYan and gluon fusion processes mediated by charged gauge bosons and gluons, respectively. A detailed study of the exotic quark production at the LHC and the exotic quark decay modes is beyond the scope of this work and is deferred for a future publication.

Furthermore, from the quark Yukawa terms of eq. (2.16), it follows that the flavor changing top quark decays $t \rightarrow h c, t \rightarrow h u$ and $t \rightarrow c Z$ are absent in our model. Besides that, the decays of charged Higgses into a SM up-type and SM down-type quarks, namely, $H_{1}^{+} \rightarrow u_{i} \bar{d}_{j}, H_{1}^{-} \rightarrow d_{i} \bar{u}_{j}, H_{2}^{+} \rightarrow u_{i} \bar{d}_{j}, H_{2}^{-} \rightarrow d_{i} \bar{u}_{j},(i, j=1,2,3)$ with $H_{1}^{ \pm}=-\rho_{1}^{ \pm}$and $H_{2}^{ \pm}=-\rho_{3}^{ \pm},\left(u_{1}, u_{2}, u_{3}\right)=(u, c, t)$ and $\left(d_{1}, d_{2}, d_{3}\right)=(d, s, b)$, are forbidden at tree level in 
our model. Out of the charged Higgs decays into SM quarks, only the decays $H_{1}^{+} \rightarrow u_{1} \bar{d}_{n}$, $H_{1}^{-} \rightarrow \overline{u_{1}} d_{n}(n=1,2)$ appear at one loop level whereas the decays $H_{1}^{+} \rightarrow u_{2} \overline{d_{n}}, H_{1}^{-} \rightarrow \overline{u_{2}} d_{n}$ $(n=1,2)$ are allowed at two loop level. In addition, the dominant SM leptonic decay modes of the charged Higgses $H_{1}^{ \pm}$and $H_{2}^{ \pm}$only appear at one loop level and correspond to the processes $H_{1}^{ \pm} \rightarrow \nu_{1} e^{ \pm}$and $H_{2}^{ \pm} \rightarrow \nu_{1}^{c} e^{ \pm}$. The remaining decay modes $H_{1}^{ \pm} \rightarrow \nu_{1} \mu^{ \pm}$, $H_{2}^{ \pm} \rightarrow \nu_{1}^{c} \mu^{ \pm}, H_{1}^{ \pm} \rightarrow \nu_{1} \tau^{ \pm}, H_{2}^{ \pm} \rightarrow \nu_{1}^{c} \tau^{ \pm}$are very tiny with respect to the decays $H_{1}^{ \pm} \rightarrow \nu_{1} e^{ \pm}$ and $H_{2}^{ \pm} \rightarrow \nu_{1}^{c} e^{ \pm}$, due to the very small mixing angles in the rotation matrix that connects the SM right handed charged leptonic fields in the interaction eigenstates with the physical SM right handed charged leptonic fields. Consequently, a measurement of the branching fraction for the $t \rightarrow h c, t \rightarrow h u, t \rightarrow c Z, H_{1}^{+} \rightarrow t \bar{d}_{j}, H_{1}^{-} \rightarrow d_{i} \bar{t}, H_{2}^{+} \rightarrow u_{i} \bar{d}_{j}, H_{2}^{-} \rightarrow d_{i} \bar{u}_{j}$ $(i, j=1,2,3), H_{1}^{ \pm} \rightarrow \nu_{1} \mu^{ \pm}, H_{2}^{ \pm} \rightarrow \nu_{1}^{c} \mu^{ \pm}, H_{1}^{ \pm} \rightarrow \nu_{1} \tau^{ \pm}, H_{2}^{ \pm} \rightarrow \nu_{1}^{c} \tau^{ \pm}$decays at the LHC will be crucial for ruling out this model.

\subsection{Tadpole cancellation mechanisms}

Notice that after $Z_{2} \times Z_{4}$ is softly broken, the terms $\bar{E}_{n L} \varphi_{1}^{0} E_{m R}$ and $\left(m_{E}\right)_{n m} \bar{E}_{n L} E_{m R}$ $(m, n=2,3)$ will generate a tadpole for $\varphi_{1}^{0}$. Since this contribution is known to give an infinite value, in order to make the theory renormalizable without giving a VEV to the $\varphi_{1}^{0}$, one has to consider also the contribution to the $\varphi_{1}^{0}$ tadpole arising from the scalar interaction $\omega_{1} \varphi_{1}^{0}\left(\varphi_{2}^{0}\right)^{2}$ with the virtual $\varphi_{2}^{0}$ in the loop. We require that these two tadpoles cancel so that $\left\langle\varphi_{1}^{0}\right\rangle=0$ be guaranteed at one-loop level. This requirement of tadpole cancellation is an ad hoc condition of viability of our model. It implies fine-tuning of the model parameters $\left(m_{E}\right)_{n m}$ and $\varphi_{1}^{0}\left(\varphi_{2}^{0}\right)^{2}$, which is unstable under the renormalization flow. In our model we do not have a symmetry to stabilize the required tadpole cancellation. Moreover, it is not possible to introduce such a symmetry without a radical modification of the model structure with all its nice features. The solution to this problem can be expected from the appropriate imbedding of our model into a more fundamental setup with additional symmetries protecting the tadpole cancelation. Given that this condition relates the parameters of the fermionic and scalar sector one may think of imbedding our model into a supersymmetric or warped five-dimensional framework (see refs. $[153,154]$ for recent reviews on extra-dimensions). Thinking of a supersymmetric (SUSY) version of our model (for some examples of SUSY 3-3-1 models see refs. [121, 131, 155-170]) we hope that even in the case of softly-broken SUSY the tadpole cancelation would be technically natural. More conservatively we may expect a violation of this cancelation not stronger than logarithms of the high-scale cutoff. In this case $\left\langle\varphi_{1}^{0}\right\rangle \neq 0$, but due to the logarithmic sensitivity to the cutoff, it would be around the electroweak scale. This is phenomenologically safe, giving rise to tree level mixing $\bar{F}_{L} f_{R}$ between an exotic, $F$, and a SM, $f$, charged fermions. Despite the presence of this mixing terms, the first and second rows of the up type quark mass matrix as well as the first three rows of the down type quark and charged lepton mass matrices will still be vanishing at tree level, which is a consequence of the symmetries of the model as well as from the fact that the $\mathrm{SU}(3)_{L}$ scalar triplet $\rho$ is inert. This implies that only the top quark and exotic fermions do acquire tree level masses, whereas the remaining SM fermions will be massless at tree level. The 
masses for the remaining SM fermions will still appear via the radiative seesaw mechanisms described in the previous subsection.

The implementation of supersymmetry or embedding our model in a warped extradimensional setup, requires careful studies, which are beyond the scope of the present paper and will be addressed elsewhere.

\section{Quark masses and mixings}

From the quark Yukawa interactions (2.16) it follows that the SM quark mass matrices are given by:

$$
M_{U}=\left(\begin{array}{ccc}
\widetilde{\varepsilon}_{11}^{(u)} & \varepsilon_{12}^{(u)} & 0 \\
\widetilde{\varepsilon}_{21}^{(u)} & \varepsilon_{22}^{(u)} & 0 \\
0 & 0 & y
\end{array}\right) \frac{v}{\sqrt{2}}, \quad \quad M_{D}=\left(\begin{array}{ccc}
\widetilde{\varepsilon}_{11}^{(d)} & \widetilde{\varepsilon}_{12}^{(d)} & \widetilde{\varepsilon}_{13}^{(d)} \\
\widetilde{\varepsilon}_{21}^{(d)} & \widetilde{\varepsilon}_{22}^{(d)} & \widetilde{\varepsilon}_{23}^{(d)} \\
\varepsilon_{31}^{(d)} & \varepsilon_{32}^{(d)} & \varepsilon_{33}^{(d)}
\end{array}\right) \frac{v}{\sqrt{2}}
$$

where $y \simeq 1$ is generated at tree level from the renormalizable Yukawa interaction $\bar{Q}_{3 L} \eta U_{3 R}$, thus giving rise to a tree level top quark mass. Furthermore, $\varepsilon_{n 2}^{(u)}(n=1,2)$ and $\varepsilon_{3 j}^{(d)}(j=$ $1,2,3)$ are dimensionless parameters generated at one loop level, whereas the dimensionless parameters $\widetilde{\varepsilon}_{n 1}^{(u)}$ and $\widetilde{\varepsilon}_{n j}^{(d)}$ arise at two loop level. The corresponding Feynman diagrams are shown in figure 1.

In what follows we will show that the SM quark mass matrices given above are consistent with the low energy quark flavor data. To this end, and considering that the $\varepsilon_{n 2}^{(u)}$ $(n=1,2)$ and $\varepsilon_{3 j}^{(d)}(j=1,2,3)$ dimensionless parameters are generated at one loop level, whereas the dimensionless parameters $\widetilde{\varepsilon}_{n 1}^{(u)}$ and $\widetilde{\varepsilon}_{n j}^{(d)}$ arise at two loop level, we choose a benchmark scenario where we set:

$$
\begin{array}{llll}
\varepsilon_{n 2}^{(u)}=a_{n 2}^{(u)} l, & \widetilde{\varepsilon}_{n 1}^{(u)}=b_{n 1}^{(u)} l^{2}, & & \\
\varepsilon_{3 j}^{(d)}=a_{3 j}^{(d)} l, & \widetilde{\varepsilon}_{n j}^{(d)}=b_{n j}^{(d)} l^{2}, & n=1,2, & j=1,2,3,
\end{array}
$$

where $l \approx(1 / 4 \pi)^{2} \approx 2.0 \times \lambda^{4}$ is the loop suppression factor and $\lambda=0.225$ is the Wolfenstein parameter. Then we expect in the model that $a_{n 2}^{(u)}, b_{n 1}^{(u)}, a_{3 j}^{(d)}, b_{n j}^{(d)}(n, m=1,2$ and $j=$ $1,2,3)$ be $\mathcal{O}(1)$ parameters.

Let us note that the large amount of independent model parameters in the fermion and scalar sectors of our model, entering in the Feynman diagrams contributing to the entries of the SM fermion mass matrices, can be absorbed in the effective parameters $\varepsilon_{n 2}^{(u)}$, $\widetilde{\varepsilon}_{n 1}^{(u)} \varepsilon_{3 j}^{(d)}, \widetilde{\varepsilon}_{n j}^{(d)}(n, m=1,2$ and $j=1,2,3)$ given by eq. (3.2). They amount to 26 real free parameters, which is a large number compared with the number of quark sector observables with the experimental values ${ }^{1}$

$$
\begin{aligned}
& m_{u}(M e V)=1.45_{-0.45}^{+0.56}, \quad m_{d}(M e V)=2.9_{-0.4}^{+0.5}, \quad m_{s}(M e V)=57.7_{-15.7}^{+16.8}, \\
& m_{c}(\mathrm{MeV})=635 \pm 86, \quad m_{t}(\mathrm{GeV})=172.1 \pm 0.6 \pm 0.9, \quad m_{b}(\mathrm{GeV})=2.82_{-0.04}^{+0.09} \text {, } \\
& \sin \theta_{12}=0.2254, \quad \sin \theta_{23}=0.0414, \quad \sin \theta_{13}=0.00355, J=2.96_{-0.16}^{+0.20} \times 10^{-5} \text {. }
\end{aligned}
$$

\footnotetext{
${ }^{1}$ We use the experimental values of the quark masses at the $M_{Z}$ scale, from ref. [171], which are similar to those in [172]. The experimental values of the CKM parameters are taken from ref. [173].
} 



Figure 1. Loop Feynman diagrams contributing to the entries of the SM quark mass matrices. Here $m, n, k=1,2$ and $j=1,2,3$, whereas $w_{1}$ corresponds to the mass dimension coefficient of the trilinear scalar coupling $\left(\left(\varphi_{2}^{0}\right)^{*}\right)^{2}\left(\varphi_{1}^{0}\right)^{*}$. The cross marks $\times$ and $\otimes$ in the internal lines correspond to the symmetry preserving and softly breaking mass insertions, respectively.

being $t, u, c, d, s, b$ quark masses, $\theta_{12}, \theta_{23}, \theta_{13}$ mixing angles and the Jarlskog parameter. Therefore, the model in its present form does not predict these observables. However, as we already commented, we only pretend to reproduce the hierarchy of the quark masses via the loop suppression predicted by the model and expressed by eq. (3.2). To wit, we consider the mass matrices (3.1) with the hierarchical matrix elements (3.2) predicted by the model. For these matrices we look for the eigenvalue problem solutions reproducing the values in eq. (3.3), under the condition that $a^{(u, d)}, b^{(u, d)}$ be most close to $\mathcal{O}(1)$. Applying the standard procedure we find a solution

$$
\begin{aligned}
& a_{12}^{(u)} \simeq a_{22}^{(u)} \approx 0.5, \quad b_{11}^{(u)} \approx 0.25, \quad b_{21}^{(u)} \approx 0.7, \\
& a_{31}^{(d)} \simeq-1.6, \quad a_{32}^{(d)} \simeq-2.2, \quad a_{33}^{(d)} \simeq 1.6, \\
& b_{11}^{(d)} \simeq-12.3-0.7 i, \quad b_{12}^{(d)} \simeq-7.6-1.0 i, \quad b_{13}^{(d)} \simeq 11.6+0.7 i, \\
& b_{21}^{(d)} \simeq-14.3+0.7 i, \quad b_{22}^{(d)} \simeq-5.6+1.0 i, \quad b_{23}^{(d)} \simeq 14.8-0.7 i .
\end{aligned}
$$

The above values reproduce exactly the central values in (3.3). The absolute values of the parameters in the first two rows are $\mathcal{O}(1)$. The values of the remaining two-loop level parameters are around $\sim 10$, but this is still within the ballpark of the same loop level, 
since the loop-suppression factor is $l \sim 10^{-2}$ (see eq. (3.2)). Thus the model reproduces fairly well the hierarchical structure of the observable quark mass spectrum as a result of the sequential loop suppression mechanism, where the top quark mass is generated at tree level, the masses for the bottom and charm quarks arise at one loop level and the light up, down and strange quarks get their masses at two loop level.

\section{Lepton masses and mixings}

The charged lepton masses are generated by the charged lepton Yukawa terms in eq. (2.16) via the loop diagrams shown in figure 2. The corresponding charged lepton mass matrix takes the form:

$$
M_{l}=\left(\begin{array}{ccc}
\widetilde{\varepsilon}_{11}^{(l)} & \varepsilon_{12}^{(l)} & \varepsilon_{13}^{(l)} \\
0 & \varepsilon_{22}^{(l)} & \varepsilon_{23}^{(l)} \\
0 & \varepsilon_{32}^{(l)} & \varepsilon_{33}^{(l)}
\end{array}\right) \frac{v}{\sqrt{2}}
$$

where $\varepsilon_{j n}^{(l)}(n=2,3$ and $j=1,2,3)$ are dimensionless parameters generated at one loop level, whereas the dimensionless parameter $\widetilde{\varepsilon}_{11}^{(l)}$ arises at two loop level. In order to express the loop order suppression explicitly we define new parameters

$$
\varepsilon_{j 3}^{(l)}=a_{j 3}^{(l)} \cdot l, \quad \varepsilon_{j 2}^{(l)}=a_{j 2}^{(l)} \cdot l, \quad \widetilde{\varepsilon}_{11}^{(l)}=a_{11}^{(l)} \cdot l^{2}, \quad j=1,2,3 .
$$

Here $l=(1 / 4 \pi)^{2} \approx 2.0 \times \lambda^{4}$ is the loop suppression factor introduced after eq. (3.2). Having 7 complex parameters, the model does not pretend to predict the charged lepton masses, but only reproduce the observed mass hierarchy. Therefore, again, as in the quark sector, we are looking for values of the $a^{(l)}$ parameters so that on one hand they reproduce the observable central values of the charged lepton masses $m_{e}=0.487 \mathrm{MeV}, m_{\mu}=102.8 \mathrm{MeV}$, $m_{\tau}=1.75 \mathrm{GeV}$ and on the other hand the condition $\left|a^{(l)}\right| \sim \mathcal{O}(1)$ is achieved as close as possible. A benchmark point in the model parameter space of this kind is

$$
a_{11}^{(l)} \approx a_{22}^{(l)} \approx-0.2, \quad a_{12}^{(l)} \approx a_{32}^{(l)} \approx-0.14, \quad a_{13}^{(l)} \approx a_{23}^{(l)} \approx a_{33}^{(l)} \approx 1.1 .
$$

All the values are not unnaturally small compared to the loop hierarchy (2-loop level)/(1loop level) $\sim l \approx 6.3 \times 10^{-3}$. Thus, as in the quark sector, the model proves to be able to reproduce the observed charged lepton mass hierarchy by a sequential loop suppression.

From the neutral lepton Yukawa interactions in eq. (2.16) we find the neutral lepton mass terms:

$$
-L_{\text {gmass }}^{(\nu)}=\frac{1}{2}\left(\overline{\nu_{L}^{C}} \overline{\nu_{R}} \overline{N_{R}}\right) M_{\nu}\left(\begin{array}{c}
\nu_{L} \\
\nu_{R}^{C} \\
N_{R}^{C}
\end{array}\right)+m_{\Psi} \overline{\Psi_{R}^{c}} \Psi_{R}+h . c,
$$

where the neutrino mass matrix $M_{\nu}$

$$
M_{\nu}=\left(\begin{array}{ccc}
M_{1} & 0_{3 \times 3} & M_{3} \\
0_{3 \times 3} & M_{2} & M_{4} \\
M_{3} & M_{4} & \mathcal{M}
\end{array}\right)
$$





Figure 2. Loop Feynman diagrams contributing to the entries of the charged lepton mass matrices. Here $k, p, r, s=2,3$ and $w_{1}$ corresponds to the mass dimension coefficients of the trilinear scalar coupling $\left(\left(\varphi_{2}^{0}\right)^{*}\right)^{2}\left(\varphi_{1}^{0}\right)^{*}$. The cross marks in the internal lines denote the $m_{E}$ mass insertions from eq. (2.18).

is generated by the loop diagrams shown in figure 3. The sub-matrices $M_{1}, M_{2}, M_{3}, M_{4}$ and $\mathcal{M}$ are given by

$$
\begin{aligned}
& M_{1}=\left(\begin{array}{ccc}
0 & a_{12} & a_{13} \\
a_{12} & a_{22} & a_{23} \\
a_{13} & a_{23} & a_{33}
\end{array}\right), \quad M_{2}=\left(\begin{array}{ccc}
0 & b_{12} & b_{13} \\
b_{12} & b_{22} & b_{23} \\
b_{13} & b_{23} & b_{33}
\end{array}\right), \\
& M_{3}=\left(\begin{array}{ccc}
\epsilon_{11} & \epsilon_{12} & \epsilon_{13} \\
\epsilon_{21} & \epsilon_{22} & \epsilon_{23} \\
\epsilon_{31} & \epsilon_{32} & \epsilon_{33}
\end{array}\right) \frac{v}{\sqrt{2}}, \quad M_{4}=\left(\begin{array}{lll}
\varepsilon_{1} & \varepsilon_{2} & \varepsilon_{3} \\
d_{1} & d_{2} & d_{3} \\
d_{4} & d_{5} & d_{6}
\end{array}\right) \frac{v_{\chi}}{\sqrt{2}}, \quad \mathcal{M}=\left(\begin{array}{lll}
\mathcal{M}_{11} & \mathcal{M}_{12} & \mathcal{M}_{13} \\
\mathcal{M}_{12} & \mathcal{M}_{22} & \mathcal{M}_{23} \\
\mathcal{M}_{13} & \mathcal{M}_{23} & \mathcal{M}_{33}
\end{array}\right) \text {, }
\end{aligned}
$$

where the matrix elements $d_{l}(l=1,2, \cdots, 6)$ arise at tree level, $\epsilon_{i j}, \varepsilon_{j}$ and $\mathcal{M}_{i j}(i, j=$ $1,2,3)$ at one loop level, whereas $a_{n m}, a_{1 n}, b_{1 n}$ and $b_{n m}(n, m=2,3)$ arise at two loop level. Let us note that $a_{1 n}$ and $b_{1 n}$ are generated by the $h_{\rho 11}^{(L)}$ and $h_{\rho n m}^{(L)}$ terms in eq. (2.16). These terms give rise to the four Feynman diagrams shown in the last two lines of figure 3. Consequently, the light active neutrino masses are generated by a combination of linear and inverse seesaw mechanisms at two loop level. 

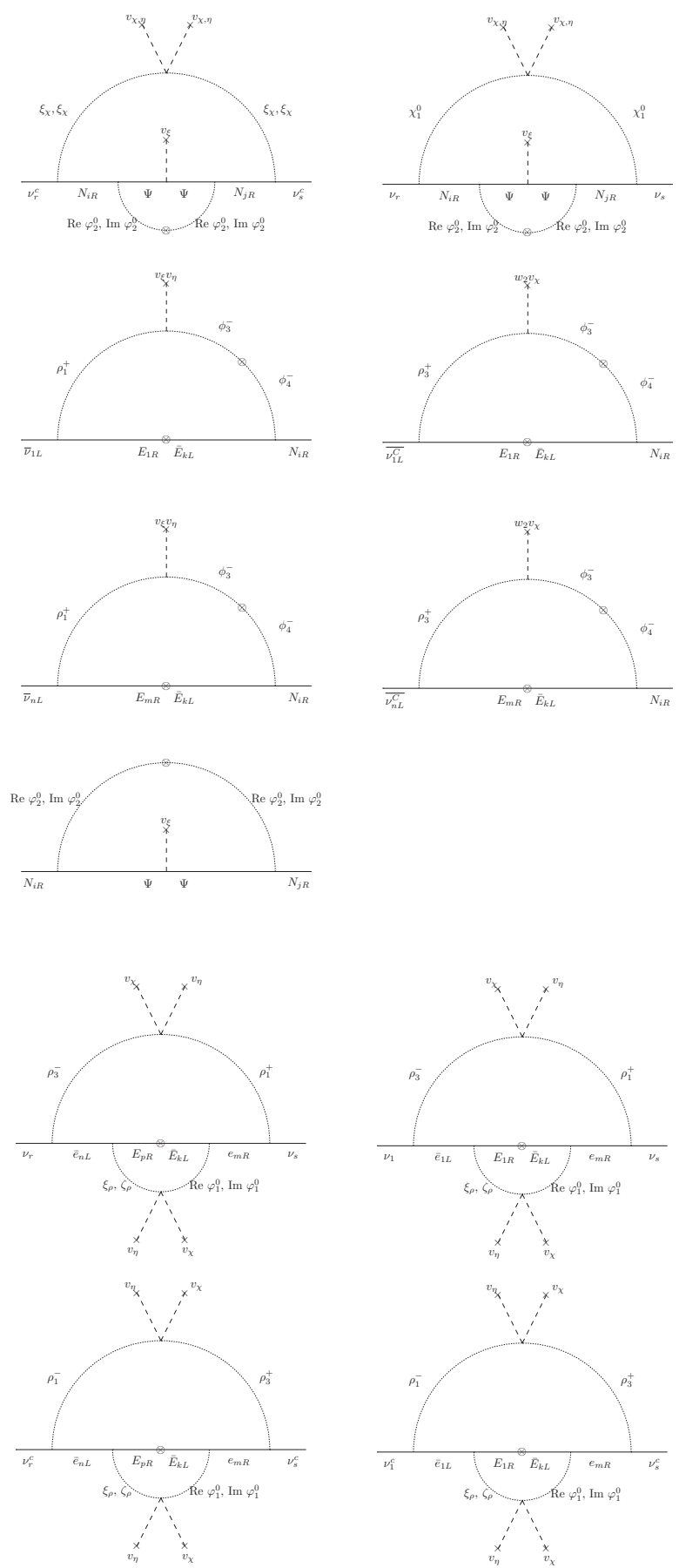

Figure 3. Loop Feynman diagrams contributing to the entries of the neutrino mass matrix. Here $n, m, k, p, r, s=2,3$ and $i, j=1,2,3$, whereas $w_{2}$ corresponds to the mass dimension coefficient of the trilinear scalar coupling $\phi_{3}^{+} \rho^{\dagger} \chi$. The cross mark $\otimes$ in the internal lines correspond to the softly breaking mass insertions. 
By performing the perturbative block diagonalization of the $9 \times 9$ neutrino mass matrix $M_{\nu}$ of eq. (4.5), which is shown in appendix B, we find that the light active neutrino mass matrix has the form:

$$
\begin{aligned}
M_{1 \nu}= & M_{1}+\frac{1}{16} M_{3}\left(M_{4}\right)^{-2} M_{3}^{T} M_{3}\left(M_{4}\right)^{-1} M_{3}^{T} M_{3}\left(M_{4}\right)^{-2} M_{3}^{T} \\
& +\frac{1}{8} M_{3}\left(M_{4}\right)^{-1}\left(\mathcal{M}-M_{3}^{T} M_{3}\left(M_{4}\right)^{-1}\right)\left(M_{4}\right)^{-1}\left(\mathcal{M}-\left(M_{4}\right)^{-1} M_{3}^{T} M_{3}\right)\left(M_{4}\right)^{-1} M_{3}^{T}
\end{aligned}
$$

whereas the sterile neutrino mass matrices are given by:

$$
\begin{aligned}
& M_{2 \nu}=-M_{4} \\
& M_{3 \nu}=M_{4}+\frac{1}{\sqrt{2}}\left(M_{3}^{T} M_{3}\left(M_{4}\right)^{-1}+\left(M_{4}\right)^{-1} M_{3}^{T} M_{3}\right) .
\end{aligned}
$$

Let us analyze eq. (4.8) and see what are the typical mass scales of the model, which allow us to reproduce the neutrino mass scale of $m_{\nu} \sim 50 \mathrm{meV}$. The non-zero matrix elements of $M_{1}$ are determined by the 2-loop diagrams in figure 3. For the benchmark region where $m_{\Psi}=y_{\Psi} v_{\xi} \gg m_{\phi_{2}^{0}}, \mu_{1}$ their contribution is

$$
a_{i j} \sim \alpha_{1}\left(\frac{1}{4 \pi}\right)^{2}\left(\frac{v_{\chi}}{v}\right)^{2} \frac{\mu_{1}^{2}}{m_{\Psi}} \log \left(m_{\Psi} / m_{\phi_{2}^{0}}\right) .
$$

This 2-loop-level contribution has typical inverse or linear seesaw structure proportional to the soft symmetry breaking parameter $\mu_{1}^{2}$. This parameter is stable against radiative corrections due to the model symmetries and, therefore, any of its possible values is technically natural. Then we can choose it arbitrarily small to adjust the observable neutrino mass scale $m_{\nu} \sim 50 \mathrm{meV}$. Note that $a_{i j} \rightarrow 0$ in the limit $m_{\Psi} \rightarrow 0$, since $m_{\Psi}$ is the only Lepton Number Violating parameter in our model.

The second term in eq. (4.8) is of the order

$$
\text { (2nd term in eq. }(4.8)) \sim\left(\frac{v}{v_{\chi}}\right)^{5} v .
$$

From the condition $\left(M_{1 \nu}\right)_{i j} \lesssim m_{\nu} \sim 50 \mathrm{meV}(i, j=, 1,2,3)$, we find that the scale of the first stage of symmetry breaking (2.1) is limited to

$$
v_{\chi} \gtrsim 90 \mathrm{TeV}
$$

It is worth mentioning that, as follows from eqs. (4.8) and (4.8), the physical neutrino eigenstates include three active neutrinos and six exotic neutrinos. Due to the structure of $M_{3,4}$ in eq. (4.6) and using eq. (4.8), it is shown in appendix $\mathrm{C}$ that the second and third generation of exotic neutrinos arising from $M_{2 \nu}$ and $M_{3 \nu}$ have $\mathcal{O}(10) \mathrm{TeV}$ scale masses, whereas the first generation ones from $M_{2 \nu}$ and $M_{3 \nu}$ have masses at the electroweak symmetry breaking scale. The $\mathcal{O}(10) \mathrm{TeV}$ scale exotic neutrinos of $M_{2 \nu}$ have a small splitting of $\sim \frac{v^{2}}{v_{\chi}}$ with respect to the ones of $M_{3 \nu}$, as indicated by eq. (4.8). These heavy quasi Dirac neutrinos can be produced in pairs at the LHC, via a Drell-Yan mechanism, mediated by a heavy non Standard Model neutral gauge boson $Z^{\prime}$. The heavy quasi Dirac neutrinos 
can decay into a Standard Model charged lepton and $W$ gauge boson, due to their mixings with the light active neutrinos. Thus, the observation of an excess of events in the dilepton final states with respect to the SM background at the LHC would be a signal supporting this model. Studies of inverse seesaw neutrino signatures at the LHC and ILC as well as the production of Heavy neutrinos at the LHC are performed in refs. [174, 175]. A detailed study of the collider phenomenology of this model is beyond the scope of the present paper and is left for future studies.

\section{Discussions and conclusions}

We have built the first renormalizable extension of the 3-3-1 model with $\beta=-\frac{1}{\sqrt{3}}$, which explains the SM fermion mass hierarchy by a sequential loop suppression. Our model, based on the 3-3-1 symmetry extended with the $\mathrm{U}(1)_{L_{g}} \times Z_{4} \times Z_{2}$ group is consistent with the low energy fermion flavor data. In the model only the top quark and the charged exotic fermions acquire tree level masses, whereas the remaining SM fermions get their masses via radiative corrections: 1 loop bottom, charm, tau and muon masses; 2-loop masses for the light up, down, strange quarks as well as for the electron. Furthermore, the light active neutrinos acquire their masses from a combination of linear and inverse seesaw mechanisms at two loop level. In our model the quark and lepton mixings arise from radiative effects. At tree level there is no quark mixing, the mixing angles in the quark sector are generated from a combination of one and two loop level radiative seesaw mechanisms. In the lepton sector, the contribution to the leptonic mixing angles coming from the charged leptons arise at one loop level, whereas the mixings in the light active neutrino sector are generated from a two loop level radiative seesaw mechanism.

Furthermore our model predicts the absence of the decays $t \rightarrow h c, t \rightarrow h u, t \rightarrow c Z$, $H_{1}^{+} \rightarrow t \bar{d}_{j}, H_{1}^{-} \rightarrow d_{i} \bar{t}, H_{2}^{+} \rightarrow u_{i} \bar{d}_{j}, H_{2}^{-} \rightarrow d_{i} \bar{u}_{j}(i, j=1,2,3), H_{1}^{ \pm} \rightarrow \nu_{1} \mu^{ \pm}, H_{2}^{ \pm} \rightarrow \nu_{1}^{c} \mu^{ \pm}$, $H_{1}^{ \pm} \rightarrow \nu_{1} \tau^{ \pm}, H_{2}^{ \pm} \rightarrow \nu_{1}^{c} \tau^{ \pm}$, which implies that a measurement of the branching fraction for these decays at the LHC will be crucial for ruling out the model. Consequently, charged Higgses can be searched at the LHC through the their decay into a SM up-type (downtype) and a exotic SM down-type $B\left(\widetilde{T}_{k}(k=1,2)\right.$ up-type $)$ quarks, as well as into a exotic charged lepton and neutrino. Since the heavy exotic $\mathrm{SU}(3)_{L}$ singlet down (up) type quark(s), i.e., $B\left(\widetilde{T}_{n}(n=1,2)\right)$ will decay predominantly into a SM down (up) type quark and the $\operatorname{Re} \varphi_{1}^{0}$ or $\operatorname{Im} \varphi_{1}^{0}$ neutral scalar (which is identified as missing energy, due to the preserved $Z_{4}$ symmetry), it follows that the observation of an excess of events with respect to the SM background in the dijet final states at the LHC can be a signal of charged Higgs decays of this model. Finally, it is worth mentioning that since charged exotic fermions are produced in pairs, and they predominantly decay into a SM charged fermion and a electrically neutral $Z_{4}$ charged scalar (identified as a missing energy), observing an excess of events with respect to the SM background in the dijet and opposite sign dilepton final states at the LHC can be a signal in support of this model. A detailed study of the exotic charged fermion production at the LHC and the exotic charged fermion decay modes is beyond the scope of this work and is deferred for a future publication. 
The final remark deals with the possible DM candidates in our model, which could be either the right handed Majorana neutrinos $N_{i R}(i=1,2,3), \Psi_{R}$, or the lightest scalars $\varphi_{1}^{R} \equiv \operatorname{Re} \varphi_{1}^{0}, \varphi_{1}^{I} \equiv \operatorname{Im} \varphi_{1}^{0}$ as well as $\varphi_{2}^{R} \equiv \operatorname{Re} \varphi_{2}^{0}, \varphi_{2}^{I} \equiv \operatorname{Im} \varphi_{2}^{0}$. Let us note that the masses $m_{1}^{R, I}, m_{2}^{R}$ of the scalars $\varphi_{1}^{R, I}, \varphi_{2}^{R}$ and the fermion $\Psi_{R}$ mass $m_{\Psi}$ are arbitrary parameters, since the corresponding mass terms are compatible with all the symmetries of the model, while $\varphi_{2}^{I}$ squared mass is $\left(m_{2}^{R}\right)^{2}-4 \mu_{1}^{2}$. The mass splitting parameter $\mu_{1}^{2}$ is the soft $Z_{4}$ breaking mass (2.19), which already showed up in the light neutrino sector (4.9). Since the light neutrino mass scale should be small, then the $\varphi_{2}^{R}-\varphi_{2}^{I}$ mass splitting should not be very large. A superficial survey shows that $N_{i R}$ could be a DM candidate only in a rather restricted domain of the model parameter space, due to the presence of $N_{R}-\nu_{L}$-mixing $U_{\nu N}$ at least at one-loop level (fifth diagram in figure 3). As a result, there is the SM charged current decay $N_{R} \rightarrow e_{L}^{-} \nu_{1} e_{R}^{+}$. The requirement that the DM lifetime be greater than the universe lifetime sets stringent constraints on $U_{\nu N}$. We do not analyze the impact of this constraint on the model parameter space and the possible correlations of the DM and the light neutrino sectors. Instead we consider the other more viable DM candidates. The gauge group singlet $\Psi_{R}$ is one of them, if its mass satisfies the condition $m_{\Psi}<m_{2}^{R}$, and then, as follows from eq. (2.16), it does not decay at tree level. Assuming that our model be valid only up to some high-energy scale $\Lambda \gg v_{\chi}$, we have to consider the possible non-renormalizable operators induced by the physics beyond this scale. It is easy to check that all such operators compatible with the symmetry group $\mathcal{G}$ of our model involve the exotic scalar $\varphi_{2}^{0}$. The lowest dimensional operator is

$$
\frac{1}{\Lambda^{3}}\left(\bar{L} \Psi_{R}\right)\left(\bar{e}_{R} L\right) \varphi_{2}^{0}
$$

Therefore, with the condition $m_{\Psi}<m_{2}^{\Psi}$ the non-renormalizable operators do not lead to kinematically allowed decays of $\Phi_{R}$, making it a stable DM particle. There is also a viable scalar DM candidate $\varphi^{0}$ in our model. This is the lightest of the exotic scalars $\operatorname{Re} \varphi_{1,2}^{0}$, $\operatorname{Im} \varphi_{1,2}^{0}$, which is also lighter than the exotic charged fermions, as well as lighter than $\Psi_{R}$, and then, as follows from eqs. (2.16), (2.16), its tree-level decays are kinematically forbidden. However, as before, we check the possible non-renormalizable operators originating from the scales $\Lambda$, above the theoretical validity of our model. In the case of the DM candidate $\varphi^{0} \equiv \operatorname{Re} \varphi_{1}^{0}$ or $\operatorname{Im} \varphi_{1}^{0}$, we find the dominant operator

$$
\frac{1}{\Lambda^{2}} \epsilon_{a b c}\left(\eta^{\dagger}\right)^{a}\left(\chi^{\dagger}\right)^{b} \varphi_{1}^{0} \bar{L}_{1}^{c} e_{k R} \quad \text { for } \quad k=2,3
$$

compatible with all the symmetries of our model. This operator induces the decays $\varphi^{0} \rightarrow$ $e_{1}^{+} e_{2,3}^{-} \xi_{\eta}, \varphi^{0} \rightarrow e_{1}^{+} e_{2,3}^{-} \zeta_{\eta}, \varphi^{0} \rightarrow e_{1}^{+} e_{2,3}^{-} \xi_{\chi}, \varphi^{0} \rightarrow e_{1}^{+} e_{2,3}^{-} \zeta_{\chi}, \varphi^{0} \rightarrow e_{1}^{+} e_{2,3}^{-}$, respectively. Here $\xi_{\eta}=\cos \alpha h^{0}+\sin \alpha H_{1}^{0}, \zeta_{\eta} \simeq G_{1}^{0}$, with $\tan \theta \sim \mathcal{O}\left(\frac{v}{v_{\chi}}\right)$ and $\alpha$ a mixing angle, which depends on the scalar potential parameters. Furthermore, $h$ is the $126 \mathrm{GeV} \mathrm{SM} \mathrm{Higgs} \mathrm{boson,} H_{1}^{0}$ is one of the physical heavy neutral Higges, whereas $G_{1}^{0}$ is the Goldstone boson associated with the longitudinal component of the $Z$ gauge boson. For the scenario where the scalar $\varphi^{0}$ is heavier than the $126 \mathrm{GeV}$ Higgs, the partial decay rates of the kinematically allowed 
processes can be estimated as

$$
\begin{aligned}
& \Gamma\left(\varphi^{0} \rightarrow Z e_{1}^{+} e_{2,3}^{-}\right) \simeq \Gamma\left(\varphi^{0} \rightarrow \zeta_{\eta} e_{1}^{+} e_{2,3}^{-}\right) \sim \Gamma\left(\varphi^{0} \rightarrow h e_{1}^{+} e_{2,3}^{-}\right) \sim m_{\varphi^{0}}^{3} \frac{v_{\chi}^{2}}{\Lambda^{4}}, \\
& \Gamma\left(\varphi^{0} \rightarrow e_{1}^{+} e_{2,3}^{-}\right) \sim m_{\varphi^{0}}\left(\frac{v_{\chi} v_{\eta}}{\Lambda^{2}}\right)^{2} .
\end{aligned}
$$

Requiring that the DM candidate $\varphi^{0}$ lifetime be greater than the universe lifetime $\tau_{u} \approx 13.8$ Gyr, taking into account the limit (4.11) and assuming $m_{\varphi^{0}} \sim 1 \mathrm{TeV}$, we estimate the cutoff scale of our model

$$
\Lambda>3 \times 10^{10} \mathrm{GeV} .
$$

Thus we conclude that under the above specified conditions the model contains viable fermionic $\Psi_{R}$ and scalar $\varphi^{0}$ DM candidates. A detailed study of the dark matter constraints in our model is beyond the scope of the present paper and will be considered elsewhere.

\section{Acknowledgments}

This research has received funding from Fondecyt (Chile) grants No. 1170803, No. 1150792, No. 1180232, No. 3150472 and by CONICYT (Chile) Ring ACT1406, PIA/Basal FB0821, the UTFSM grant FI4061, the Vietnam National Foundation for Science and Technology Development (NAFOSTED) under Grant No. 103.01-2017.356. A.E.C.H is very grateful to the Institute of Physics, Vietnam Academy of Science and Technology for the warm hospitality and for fully financing his visit.

\section{A Generalized lepton number}

Since the lepton and anti-lepton lie in the triplet, the lepton number operator $L$ does not commute with the $\mathrm{SU}(3)_{C} \times \mathrm{SU}(3)_{L} \times \mathrm{U}(1)_{X}$ gauge symmetry and has the form [127]

$$
L=\frac{4}{\sqrt{3}} T_{8}+L_{g}=\left(\begin{array}{ccc} 
\pm \frac{2}{3}+L_{g} & 0 & 0 \\
0 & \pm \frac{2}{3}+L_{g} & 0 \\
0 & 0 & \mp \frac{4}{3}+L_{g}
\end{array}\right)
$$

where the upper and lower signs correspond to triplet and antitriplet of $\mathrm{SU}(3)_{L}$, respectively. Here $L_{g}$ is a conserved charge corresponding to the $\mathrm{U}(1)_{L_{g}}$ global symmetry, which commutes with the gauge symmetry. According to the analysis done in ref. [127], the $\mathrm{SU}(3)_{L}$ Higgs triplets $\chi$ and $\eta$ have different $L_{g}$ charges, which are given by:

$$
L_{g}(\chi)=\frac{4}{3}, \quad L_{g}(\eta)=-\frac{2}{3} .
$$

From the application of (A.1) to (2.8), it follows that the top component of $\chi$ and the bottom one of $\eta$ carry lepton number $L\left(\chi_{1}^{0}\right)=-L\left(\eta_{3}^{0}\right)=2$ whereas the other components do not $L\left(\chi_{3}^{0}\right)=L\left(\eta_{1}^{0}\right)=0$. 


\section{B Perturbative diagonalization of the neutrino mass matrix}

In this appendix we show explicitly the perturbative diagonalization of the $9 \times 9$ neutrino mass matrix $M_{\nu}$ of our model, which is given by eqs. (4.4)-(4.6). The elements of the submatrices $M_{1,2,3,4}$ obey the following hierarchy:

$$
\left(M_{1}\right)_{i j} \sim\left(M_{2}\right)_{i j} \ll \mathcal{M}_{i j} \ll\left(M_{3}\right)_{i j} \ll\left(M_{4}\right)_{i j}
$$

with $i, j=1,2,3$.

We first apply the following orthogonal transformation to the matrix $M_{\nu}$ :

$$
S_{\nu}^{T} M_{\nu} S_{\nu} \simeq\left(\begin{array}{ccc}
M_{1} & \frac{1}{\sqrt{2}} M_{3} & \frac{1}{\sqrt{2}} M_{3} \\
\frac{1}{\sqrt{2}} M_{3}^{T} & M_{4} & \frac{1}{2} \mathcal{M} \\
\frac{1}{\sqrt{2}} M_{3}^{T} & \frac{1}{2} \mathcal{M} & -M_{4}
\end{array}\right), \quad S_{\nu}=\left(\begin{array}{ccc}
I & 0 & 0 \\
0 & \frac{1}{\sqrt{2}} I & \frac{1}{\sqrt{2}} I \\
0 & -\frac{1}{\sqrt{2}} I & \frac{1}{\sqrt{2}} I
\end{array}\right)
$$

where $I$ is the $3 \times 3$ identity matrix.

Then, a second orthogonal transformation is applied under the matrix $M_{\nu}$, as follows:

$$
\begin{aligned}
& R_{1 \nu}^{T} S_{\nu}^{T} M_{\nu} S_{\nu} R_{1 \nu} \simeq \\
& \left(\begin{array}{ccc}
M_{1}+\frac{1}{\sqrt{2}} M_{3} B_{1}^{T}+\frac{1}{\sqrt{2}} B_{1} M_{3}^{T}-B_{1} M_{4} B_{1}^{T} & \frac{1}{\sqrt{2}} M_{3}+\frac{1}{2} B_{1} \mathcal{M} & \frac{1}{\sqrt{\sqrt{2}}} M_{3}-B_{1} M_{4} \\
\frac{1}{\sqrt{2}} M_{3}^{T}+\frac{1}{2} \mathcal{M} B_{1}^{T} & M_{4} & \frac{1}{2} \mathcal{M}-\frac{1}{\sqrt{2}} M_{3}^{T} B_{1} \\
\frac{1}{\sqrt{2}} M_{3}^{T}-M_{4} B_{1}^{T} & \frac{1}{2} \mathcal{M}-\frac{1}{\sqrt{2}} B_{1}^{T} M_{3}-M_{4}-\frac{1}{\sqrt{2}} M_{3}^{T} B_{1}-\frac{1}{\sqrt{2}} B_{1}^{T} M_{3}+B_{1}^{T} M_{1} B_{1}
\end{array}\right)
\end{aligned}
$$

where the rotation matrix $R_{1 \nu}$ is given by:

$$
R_{1 \nu}=\left(\begin{array}{ccc}
1-\frac{1}{2} B_{1} B_{1}^{T} & 0 & -B_{1} \\
0 & 1 & 0 \\
B_{1}^{T} & 0 & 1-\frac{1}{2} B_{1} B_{1}^{T}
\end{array}\right)
$$

The partial diagonalization condition:

$$
\left(R_{1 \nu}^{T} S_{\nu}^{T} M_{\nu} S_{\nu} R_{1 \nu}\right)_{n m}=\left(R_{1 \nu}^{T} S_{\nu}^{T} M_{\nu} S_{\nu} R_{1 \nu}\right)_{m n}=0, \quad n=1,2,3 \quad m=7,8,9 .
$$

yields the following relation:

$$
B_{1} \simeq \frac{1}{\sqrt{2}} M_{3}\left(M_{4}\right)^{-1}
$$

Thus, eq. (B.3) takes the form:

$$
R_{1 \nu}^{T} S_{\nu}^{T} M_{\nu} S_{\nu} R_{1 \nu} \simeq\left(\begin{array}{ccc}
M_{1}+\frac{1}{2} M_{3}\left(M_{4}\right)^{-1} M_{3}^{T} & \frac{1}{\sqrt{2}} M_{3} & 0 \\
\frac{1}{\sqrt{2}} M_{3}^{T} & M_{4} & \frac{1}{2} \mathcal{M}-\frac{1}{2} M_{3}^{T} M_{3}\left(M_{4}\right)^{-1} \\
0 & \frac{1}{2} \mathcal{M}-\frac{1}{2}\left(M_{4}\right)^{-1} M_{3}^{T} M_{3} & -M_{4}
\end{array}\right)
$$

Now, a third orthogonal transformation is applied under the matrix $M_{\nu}$ :

$$
\begin{aligned}
& R_{2 \nu}^{T} R_{1 \nu}^{T} S_{\nu}^{T} M_{\nu} S_{\nu} R_{1 \nu} R_{2 \nu} \simeq \\
& (\mathrm{B} .8) \\
& \left(\begin{array}{ccc}
f_{c}\left(M_{1}+\frac{1}{2} M_{3}\left(M_{4}\right)^{-1} M_{3}^{T}\right) f_{c}+\frac{1}{\sqrt{2}} f_{c} M_{3} B_{2}^{T}+\frac{1}{\sqrt{2}} B_{2} M_{3}^{T} f_{c}+B_{2} M_{4} B_{2}^{T} & \frac{1}{\sqrt{2}} M_{3}+B_{2} M_{4} & \frac{1}{2} B_{2} \mathcal{M}-\frac{1}{2} B_{2} M_{3}^{T} M_{3}\left(M_{4}\right)^{-1} \\
\frac{1}{\sqrt{2}} M_{3}^{T}+M_{4} B_{2}^{T} & M_{4}-\frac{1}{\sqrt{2}}\left(M_{3}^{T} B_{2}+B_{2}^{T} M_{3}\right) & \frac{1}{2} \mathcal{M}-\frac{1}{2} M_{3}^{T} M_{3}\left(M_{4}\right)^{-1} \\
\frac{1}{2} \mathcal{M} B_{2}^{T}-\frac{1}{2}\left(M_{4}\right)^{-1} M_{3}^{T} M_{3} B_{2}^{T} & \frac{1}{2} \mathcal{M}-\frac{1}{2}\left(M_{4}\right)^{-1} M_{3}^{T} M_{3} & -M_{4}
\end{array}\right)
\end{aligned}
$$


where

$$
f_{s}=B_{2}, \quad f_{c}=1-\frac{1}{2} B_{2} B_{2}^{T},
$$

and the rotation matrix $R_{2 \nu}$ is given by:

$$
R_{2 \nu}=\left(\begin{array}{clc}
1-\frac{1}{2} B_{2} B_{2}^{T} & 0 & -B_{2} \\
0 & 1 & 0 \\
B_{2}^{T} & 0 & 1-\frac{1}{2} B_{2} B_{2}^{T}
\end{array}\right)
$$

The resulting partial diagonalization condition:

$$
\left(R_{2 \nu}^{T} R_{1 \nu}^{T} S_{\nu}^{T} M_{\nu} S_{\nu} R_{1 \nu} R_{2 \nu}\right)_{n m}=\left(R_{2 \nu}^{T} R_{1 \nu}^{T} S_{\nu}^{T} M_{\nu} S_{\nu} R_{1 \nu} R_{2 \nu}\right)_{m n}=0, \quad n=1,2,3 m=4,5,6
$$

yields the following relation:

$$
B_{2} \simeq-\frac{1}{\sqrt{2}} M_{3}\left(M_{4}\right)^{-1}
$$

Consequently, eq. (B.8) takes the form:

$$
\begin{aligned}
& R_{2 \nu}^{T} R_{1 \nu}^{T} S_{\nu}^{T} M_{\nu} S_{\nu} R_{1 \nu} R_{2 \nu} \simeq \\
& \left(\begin{array}{ccc}
\widetilde{M}_{1} & 0 & -\frac{1}{\sqrt{2}} M_{3}\left(M_{4}\right)^{-1}\left(\frac{1}{2} \mathcal{M}-\frac{1}{2} M_{3}^{T} M_{3}\left(M_{4}\right)^{-1}\right) \\
0 & \frac{1}{2} \mathcal{M}-\frac{1}{2} M_{3}^{T} M_{3}\left(M_{4}\right)^{-1} \\
-\frac{1}{\sqrt{2}}\left(\frac{1}{2} \mathcal{M}-\frac{1}{2}\left(M_{4}\right)^{-1} M_{3}^{T} M_{3}\right)\left(M_{4}\right)^{-1} M_{3}^{T} & \frac{1}{2} \mathcal{M}-\frac{1}{2}\left(M_{4}\right)^{-1} M_{3}^{T} M_{3} & -M_{4}
\end{array}\right) \\
& =\left(\begin{array}{ccc}
W & 0 & X \\
0 & Z & Y \\
X^{T} & Y^{T} & -M_{4}
\end{array}\right)
\end{aligned}
$$

where

$$
\begin{aligned}
W & =\widetilde{M}_{1}=M_{1}+\frac{1}{16} M_{3}\left(M_{4}\right)^{-2} M_{3}^{T} M_{3}\left(M_{4}\right)^{-1} M_{3}^{T} M_{3}\left(M_{4}\right)^{-2} M_{3}^{T} \\
Z & =\widetilde{M}_{4}=M_{4}+\frac{1}{\sqrt{2}}\left(M_{3}^{T} M_{3}\left(M_{4}\right)^{-1}+\left(M_{4}\right)^{-1} M_{3}^{T} M_{3}\right) \\
X & =-\frac{1}{\sqrt{2}} M_{3}\left(M_{4}\right)^{-1}\left(\frac{1}{2} \mathcal{M}-\frac{1}{2} M_{3}^{T} M_{3}\left(M_{4}\right)^{-1}\right) \\
Y & =\frac{1}{2} \mathcal{M}-\frac{1}{2}\left(M_{4}\right)^{-1} M_{3}^{T} M_{3}
\end{aligned}
$$

Then we apply a fourth orthogonal transformation under the matrix $M_{\nu}$, as follows:

$$
\begin{aligned}
& R_{3 \nu}^{T} R_{2 \nu}^{T} R_{1 \nu}^{T} S_{\nu}^{T} M_{\nu} S_{\nu} R_{1 \nu} R_{2 \nu} R_{3 \nu} \\
& \simeq\left(\begin{array}{ccc}
W+X\left(M_{4}\right)^{-1} X^{T} & 0 & 0 \\
0 & Z & Y \\
0 & Y^{T}-M_{4}-X^{T} B_{3}-B_{3}^{T} X+B_{3}^{T} W B_{3}
\end{array}\right)
\end{aligned}
$$


where the rotation matrix $R_{3 \nu}$ is given by:

$$
R_{3 \nu}=\left(\begin{array}{ccc}
1-\frac{1}{2} B_{3} B_{3}^{T} & 0 & -B_{3} \\
0 & 1 & 0 \\
B_{3}^{T} & 0 & 1-\frac{1}{2} B_{3} B_{3}^{T}
\end{array}\right), \quad B_{3} \simeq X\left(M_{4}\right)^{-1}
$$

Consequently, the light active neutrino mass matrix takes the form:

$$
\begin{aligned}
M_{1 \nu}= & W+X\left(M_{4}\right)^{-1} X^{T} \\
= & M_{1}+\frac{1}{16} M_{3}\left(M_{4}\right)^{-2} M_{3}^{T} M_{3}\left(M_{4}\right)^{-1} M_{3}^{T} M_{3}\left(M_{4}\right)^{-2} M_{3}^{T} \\
& +\frac{1}{8} M_{3}\left(M_{4}\right)^{-1}\left(\mathcal{M}-M_{3}^{T} M_{3}\left(M_{4}\right)^{-1}\right)\left(M_{4}\right)^{-1}\left(\mathcal{M}-\left(M_{4}\right)^{-1} M_{3}^{T} M_{3}\right)\left(M_{4}\right)^{-1} M_{3}^{T}
\end{aligned}
$$

On the other hand, from eq. (B.14) and considering the hierarchy given by eq. (B.1), it follows that the matrix of eq. (B.15) is nearly block diagonal, which implies that the sterile neutrino mass matrices are given by:

$$
\begin{aligned}
& M_{2 \nu}=-M_{4} \\
& M_{3 \nu}=M_{4}+\frac{1}{\sqrt{2}}\left(M_{3}^{T} M_{3}\left(M_{4}\right)^{-1}+\left(M_{4}\right)^{-1} M_{3}^{T} M_{3}\right)=M_{4}+\Delta .
\end{aligned}
$$

\section{Sterile neutrino mass spectrum}

In this appendix we compute the sterile neutrino mass spectrum. Our starting point is the fact that the sterile neutrino mass matrices $M_{2 \nu}=-M_{4}$ and $M_{3 \nu}=M_{4}+\Delta$ satisfy the relation:

$$
M_{4} M_{4}^{T}=\left(\begin{array}{ccc}
\varepsilon_{1}^{2}+\varepsilon_{2}^{2}+\varepsilon_{3}^{2} & \varepsilon_{1} d_{1}+\varepsilon_{2} d_{2}+\varepsilon_{3} d_{3} & \varepsilon_{1} d_{4}+\varepsilon_{2} d_{5}+\varepsilon_{3} d_{6} \\
\varepsilon_{1} d_{1}+\varepsilon_{2} d_{2}+\varepsilon_{3} d_{3} & d_{1}^{2}+d_{2}^{2}+d_{3}^{2} & d_{1} d_{4}+d_{2} d_{5}+d_{3} d_{6} \\
\varepsilon_{1} d_{4}+\varepsilon_{2} d_{5}+\varepsilon_{3} d_{6} & d_{1} d_{4}+d_{2} d_{5}+d_{3} d_{6} & d_{4}^{2}+d_{5}^{2}+d_{6}^{2}
\end{array}\right) \frac{v_{\chi}^{2}}{2}
$$

where the subleading $\mathcal{O}\left(\frac{v^{2}}{v_{\chi}}\right)$ corrections presented in $M_{3 \nu}$ have been neglected. Then, from the previous expression, it follows that:

$$
\operatorname{det}\left(M_{4} M_{4}^{T}\right)=\left(\varepsilon_{1} d_{2} d_{6}-\varepsilon_{1} d_{3} d_{5}-\varepsilon_{2} d_{1} d_{6}+\varepsilon_{2} d_{3} d_{4}+\varepsilon_{3} d_{1} d_{5}-\varepsilon_{3} d_{2} d_{4}\right)^{2} \frac{v_{\chi}^{6}}{8},
$$

Since $\varepsilon_{i} \ll d_{k}(i=1,2,3$ and $k=1,2, \cdots 6)$, the mixing angles between the first generation sterile neutrinos and the second and third generation ones can be neglected, being of the order of $\frac{\varepsilon_{i}}{d_{k}}$. Consequently, the masses for the first, second and third generation sterile neutrinos are respectively given by:

$$
\begin{aligned}
& m_{1}^{(1,2)}=\frac{4\left(\varepsilon_{1} d_{2} d_{6}-\varepsilon_{1} d_{3} d_{5}-\varepsilon_{2} d_{1} d_{6}+\varepsilon_{2} d_{3} d_{4}+\varepsilon_{3} d_{1} d_{5}-\varepsilon_{3} d_{2} d_{4}\right)}{\left(r^{2}-s\right)} \frac{v_{\chi}}{\sqrt{2}}, \\
& m_{2}^{(1,2)}=\frac{1}{2}(r-\sqrt{s}) \frac{v_{\chi}}{\sqrt{2}}, \quad m_{3}^{(1,2)}=\frac{1}{2}(r+\sqrt{s}) \frac{v_{\chi}}{\sqrt{2}},
\end{aligned}
$$


where the superscripts 1 and 2 in eq. (C.3) correspond to the physical neutrino states arising from $M_{2 \nu}$ and $M_{3 \nu}$, respectively. Furthermore, $r$ and $s$ are given by:

$$
\begin{aligned}
r= & d_{1}^{2}+d_{2}^{2}+d_{3}^{2}+d_{4}^{2}+d_{5}^{2}+d_{6}^{2}, \\
s= & d_{1}^{4}+2 d_{1}^{2} d_{2}^{2}+2 d_{1}^{2} d_{3}^{2}+2 d_{1}^{2} d_{4}^{2}-2 d_{1}^{2} d_{5}^{2}-2 d_{1}^{2} d_{6}^{2}+8 d_{1} d_{2} d_{4} d_{5}+8 d_{1} d_{3} d_{4} d_{6}+d_{2}^{4}+2 d_{2}^{2} d_{3}^{2} \\
& -2 d_{2}^{2} d_{4}^{2}+2 d_{2}^{2} d_{5}^{2}-2 d_{2}^{2} d_{6}^{2}+8 d_{2} d_{3} d_{5} d_{6}+d_{3}^{4}-2 d_{3}^{2} d_{4}^{2}-2 d_{3}^{2} d_{5}^{2}+2 d_{3}^{2} d_{6}^{2}+d_{4}^{4}+2 d_{4}^{2} d_{5}^{2} \\
& +2 d_{4}^{2} d_{6}^{2}+d_{5}^{4}+2 d_{5}^{2} d_{6}^{2}+d_{6}^{4}
\end{aligned}
$$

Since $v_{\chi} \sim \mathcal{O}(10) \mathrm{TeV}, d_{k} \sim \mathcal{O}(1)$ and $\varepsilon_{i} \sim \mathcal{O}\left(10^{-2}\right)$, we find $m_{1}^{(1,2)} \sim \mathcal{O}(100) \mathrm{GeV}$ and $m_{2,3}^{(1,2)} \sim \mathcal{O}(10) \mathrm{TeV}$. This shows that the second and third generation of exotic neutrinos arising from $M_{2 \nu}$ and $M_{3 \nu}$ have $\mathcal{O}(10) \mathrm{TeV}$ scale masses, whereas the first generation ones from $M_{2 \nu}$ and $M_{3 \nu}$ have masses at the electroweak symmetry breaking scale. The $\mathcal{O}(10) \mathrm{TeV}$ scale exotic neutrinos of $M_{2 \nu}$ have a small splitting of $\sim \frac{v^{2}}{v_{\chi}}$ with the ones of $M_{3 \nu}$, as indicated by eq. (B.18).

Open Access. This article is distributed under the terms of the Creative Commons Attribution License (CC-BY 4.0), which permits any use, distribution and reproduction in any medium, provided the original author(s) and source are credited.

\section{References}

[1] ATLAS collaboration, Observation of a new particle in the search for the Standard Model Higgs boson with the ATLAS detector at the LHC, Phys. Lett. B 716 (2012) 1 [arXiv: 1207.7214] [INSPIRE].

[2] CMS collaboration, Observation of a new boson at a mass of $125 \mathrm{GeV}$ with the CMS experiment at the LHC, Phys. Lett. B $\mathbf{7 1 6}$ (2012) 30 [arXiv:1207.7235] [INSPIRE].

[3] Particle Data Group collaboration, K.A. Olive et al., Review of Particle Physics, Chin. Phys. C 38 (2014) 090001 [inSPIRE].

[4] K.M. Patel, An $\mathrm{SO}(10) \times S_{4}$ Model of Quark-Lepton Complementarity, Phys. Lett. B 695 (2011) 225 [arXiv: 1008.5061] [inSPIRE].

[5] S. Morisi, K.M. Patel and E. Peinado, Model for T2K indication with maximal atmospheric angle and tri-maximal solar angle, Phys. Rev. D 84 (2011) 053002 [arXiv:1107.0696] [INSPIRE].

[6] S. Gupta, A.S. Joshipura and K.M. Patel, Minimal extension of tri-bimaximal mixing and generalized $Z_{2} \times Z_{2}$ symmetries, Phys. Rev. D 85 (2012) 031903 [arXiv:1112.6113] [INSPIRE].

[7] P.S. Bhupal Dev, B. Dutta, R.N. Mohapatra and M. Severson, $\theta_{13}$ and Proton Decay in a Minimal SO(10) $\times S_{4}$ model of Flavor, Phys. Rev. D 86 (2012) 035002 [arXiv:1202.4012] [INSPIRE].

[8] S.F. King, S. Morisi, E. Peinado and J.W.F. Valle, Quark-Lepton Mass Relation in a Realistic $A_{4}$ Extension of the Standard Model, Phys. Lett. B 724 (2013) 68 [arXiv: 1301.7065] [INSPIRE].

[9] A. Aranda, C. Bonilla, S. Morisi, E. Peinado and J.W.F. Valle, Dirac neutrinos from flavor symmetry, Phys. Rev. D 89 (2014) 033001 [arXiv:1307.3553] [inSPIRE]. 
[10] J.C. Gómez-Izquierdo, F. González-Canales and M. Mondragon, $Q_{6}$ as the flavor symmetry in a non-minimal SUSY SU(5) model, Eur. Phys. J. C 75 (2015) 221 [arXiv:1312.7385] [INSPIRE].

[11] A.E. Carcamo Hernandez, I. de Medeiros Varzielas, S.G. Kovalenko, H. Päs and I. Schmidt, Lepton masses and mixings in an $A_{4}$ multi-Higgs model with a radiative seesaw mechanism, Phys. Rev. D 88 (2013) 076014 [arXiv:1307.6499] [INSPIRE].

[12] M.D. Campos, A.E. Cárcamo Hernández, S. Kovalenko, I. Schmidt and E. Schumacher, Fermion masses and mixings in an $\mathrm{SU}(5)$ grand unified model with an extra flavor symmetry, Phys. Rev. D 90 (2014) 016006 [arXiv:1403.2525] [InSPIRE].

[13] C. Bonilla, S. Morisi, E. Peinado and J.W.F. Valle, Relating quarks and leptons with the $T_{7}$ flavour group, Phys. Lett. B $\mathbf{7 4 2}$ (2015) 99 [arXiv:1411.4883] [INSPIRE].

[14] M.D. Campos, A.E. Cárcamo Hernández, H. Päs and E. Schumacher, Higgs $\rightarrow \mu \tau$ as an indication for $S_{4}$ flavor symmetry, Phys. Rev. D 91 (2015) 116011 [arXiv:1408.1652] [INSPIRE].

[15] C. Arbeláez, A.E. Cárcamo Hernández, S. Kovalenko and I. Schmidt, Adjoint SU(5) GUT model with $T_{7}$ flavor symmetry, Phys. Rev. D 92 (2015) 115015 [arXiv:1507.03852] [INSPIRE].

[16] A.E. Cárcamo Hernández, I. de Medeiros Varzielas and E. Schumacher, Fermion and scalar phenomenology of a two-Higgs-doublet model with $S_{3}$, Phys. Rev. D 93 (2016) 016003 [arXiv: 1509.02083] [INSPIRE].

[17] A.E. Cárcamo Hernández, I. de Medeiros Varzielas and N.A. Neill, Novel Randall-Sundrum model with $S_{3}$ flavor symmetry, Phys. Rev. D 94 (2016) 033011 [arXiv:1511.07420] [INSPIRE].

[18] A.E. Cárcamo Hernández, A novel and economical explanation for SM fermion masses and mixings, Eur. Phys. J. C 76 (2016) 503 [arXiv:1512.09092] [INSPIRE].

[19] A.S. Joshipura and K.M. Patel, Generalized $\mu-\tau$ symmetry and discrete subgroups of $O(3)$, Phys. Lett. B 749 (2015) 159 [arXiv:1507.01235] [INSPIRE].

[20] F. Björkeroth, F.J. de Anda, I. de Medeiros Varzielas and S.F. King, Towards a complete $\Delta(27) \times \mathrm{SO}(10)$ SUSY GUT, Phys. Rev. D 94 (2016) 016006 [arXiv:1512.00850] [INSPIRE].

[21] F. Björkeroth, F.J. de Anda, I. de Medeiros Varzielas and S.F. King, Towards a complete $A_{4} \times \mathrm{SU}(5)$ SUSY GUT, JHEP 06 (2015) 141 [arXiv:1503.03306] [INSPIRE].

[22] I. de Medeiros Varzielas, $\Delta(27)$ family symmetry and neutrino mixing, JHEP 08 (2015) 157 [arXiv: 1507.00338] [INSPIRE].

[23] P. Chen, G.-J. Ding, A.D. Rojas, C.A. Vaquera-Araujo and J.W.F. Valle, Warped flavor symmetry predictions for neutrino physics, JHEP 01 (2016) 007 [arXiv: 1509.06683] [INSPIRE].

[24] E. Ma, Neutrino mixing: $A_{4}$ variations, Phys. Lett. B 752 (2016) 198 [arXiv:1510.02501] [INSPIRE].

[25] E. Ma, Soft $A_{4} \rightarrow Z_{3}$ symmetry breaking and cobimaximal neutrino mixing, Phys. Lett. B 755 (2016) 348 [arXiv: 1601.00138] [INSPIRE]. 
[26] C. Arbeláez, A.E. Cárcamo Hernández, S. Kovalenko and I. Schmidt, Radiative Seesaw-type Mechanism of Fermion Masses and Non-trivial Quark Mixing, Eur. Phys. J. C 77 (2017) 422 [arXiv: 1602.03607] [INSPIRE].

[27] P. Pasquini, S.C. Chuliá and J.W.F. Valle, Neutrino oscillations from warped flavor symmetry: predictions for long baseline experiments T2K, NOvA and DUNE, Phys. Rev. D 95 (2017) 095030 [arXiv:1610.05962] [INSPIRE].

[28] B. Carballo-Perez, E. Peinado and S. Ramos-Sanchez, $\Delta(54)$ flavor phenomenology and strings, JHEP 12 (2016) 131 [arXiv:1607.06812] [INSPIRE].

[29] V.V. Vien and H.N. Long, Lepton mass and mixing in a simple extension of the Standard Model based on T7 flavor symmetry, arXiv:1609.03895 [INSPIRE].

[30] S. Centelles Chuliá, R. Srivastava and J.W.F. Valle, CP violation from flavor symmetry in a lepton quarticity dark matter model, Phys. Lett. B 761 (2016) 431 [arXiv:1606.06904] [INSPIRE].

[31] J.T. Penedo, S.T. Petcov and A.V. Titov, Neutrino mixing and leptonic CP-violation from $S_{4}$ flavour and generalised CP symmetries, JHEP 12 (2017) 022 [arXiv:1705.00309] [INSPIRE].

[32] A.A. Cruz and M. Mondragón, Neutrino masses, mixing and leptogenesis in an S3 model, arXiv: 1701.07929 [INSPIRE].

[33] V.V. Vien and H.N. Long, The $D_{4}$ flavor symmery in 3-3-1 model with neutral leptons, Int. J. Mod. Phys. A 28 (2013) 1350159 [arXiv:1312.5034] [InSPIRE].

[34] A.E. Cárcamo Hernández, R. Martinez and F. Ochoa, Fermion masses and mixings in the 3-3-1 model with right-handed neutrinos based on the $S_{3}$ flavor symmetry, Eur. Phys. J. C 76 (2016) 634 [arXiv: 1309.6567] [INSPIRE].

[35] V.V. Vien and H.N. Long, Neutrino mixing with nonzero $\theta_{13}$ and CP-violation in the 3-3-1 model based on $A_{4}$ flavor symmetry, Int. J. Mod. Phys. A 30 (2015) 1550117 [arXiv: 1405.4665] [INSPIRE].

[36] V.V. Vien and H.N. Long, The $T_{7}$ flavor symmetry in 3-3-1 model with neutral leptons, JHEP 04 (2014) 133 [arXiv:1402.1256] [InSPIRE].

[37] V.V. Vien and H.N. Long, Neutrino mass and mixing in the 3-3-1 model and $S_{3}$ flavor symmetry with minimal Higgs content, Zh. Eksp. Teor. Fiz. 145 (2014) 991 [arXiv: 1404.6119] [INSPIRE].

[38] V.V. Vien and H.N. Long, Quark masses and mixings in the 3-3-1 model with neutral leptons based on $D_{4}$ flavor symmetry, J. Korean Phys. Soc. 66 (2015) 1809 [arXiv: 1408.4333] [INSPIRE].

[39] A.E. Cárcamo Hernández, E. Cataño Mur and R. Martinez, Lepton masses and mixing in $\mathrm{SU}(3)_{C} \otimes \mathrm{SU}(3)_{L} \otimes \mathrm{U}(1)_{X}$ models with a $S_{3}$ flavor symmetry, Phys. Rev. D 90 (2014) 073001 [arXiv: 1407.5217 ] [INSPIRE].

[40] A.E. Cárcamo Hernández, R. Martinez and J. Nisperuza, $S_{3}$ discrete group as a source of the quark mass and mixing pattern in 331 models, Eur. Phys. J. C 75 (2015) 72 [arXiv: 1401.0937] [INSPIRE].

[41] V.V. Vien, H.N. Long and D.P. Khoi, Neutrino Mixing with Non-Zero $\theta_{13}$ and CP-violation in the 3-3-1 Model Based on $S_{4}$ Flavor Symmetry, Int. J. Mod. Phys. A 30 (2015) 1550102 [arXiv: 1506.06063] [INSPIRE]. 
[42] A.E. Cárcamo Hernández and R. Martinez, A predictive 3-3-1 model with $A_{4}$ flavor symmetry, Nucl. Phys. B 905 (2016) 337 [arXiv:1501.05937] [INSPIRE].

[43] A.E. Cárcamo Hernández and R. Martinez, Fermion mass and mixing pattern in a minimal T7 flavor 331 model, J. Phys. G 43 (2016) 045003 [arXiv:1501.07261] [InSPIRE].

[44] V.V. Vien, A.E. Cárcamo Hernández and H.N. Long, The $\Delta(27)$ flavor 3-3-1 model with neutral leptons, Nucl. Phys. B 913 (2016) 792 [arXiv:1601.03300] [INSPIRE].

[45] A.E. Cárcamo Hernández, H.N. Long and V.V. Vien, A 3-3-1 model with right-handed neutrinos based on the $\Delta(27)$ family symmetry, Eur. Phys. J. C 76 (2016) 242 [arXiv: 1601.05062] [INSPIRE].

[46] J.E. Camargo-Molina, A.P. Morais, A. Ordell, R. Pasechnik, M.O. Sampaio and J. Wessén, Reviving trinification models through an E6 -extended supersymmetric GUT, Phys. Rev. D 95 (2017) 075031 [arXiv: 1610.03642] [INSPIRE].

[47] J.E. Camargo-Molina, A.P. Morais, R. Pasechnik and J. Wessén, On a radiative origin of the Standard Model from Trinification, JHEP 09 (2016) 129 [arXiv:1606.03492] [INSPIRE].

[48] J.C. Gómez-Izquierdo, Non-minimal flavored $S_{3} \otimes Z_{2}$ left-right symmetric model, Eur. Phys. J. C 77 (2017) 551 [arXiv:1701.01747] [inSPIRE].

[49] J.C. Gómez-Izquierdo, F. Gonzalez-Canales and M. Mondragón, On $\mathbf{Q}_{6}$ flavor symmetry and the breaking of $\mu \leftrightarrow \tau$ symmetry, Int. J. Mod. Phys. A 32 (2017) 1750171 [arXiv: 1705.06324] [INSPIRE].

[50] P. Chattopadhyay and K.M. Patel, Discrete symmetries for electroweak natural type-I seesaw mechanism, Nucl. Phys. B 921 (2017) 487 [arXiv:1703.09541] [INSPIRE].

[51] A.E. Cárcamo Hernández and H.N. Long, A highly predictive $A_{4}$ flavour 3-3-1 model with radiative inverse seesaw mechanism, J. Phys. G 45 (2018) 045001 [arXiv:1705.05246] [INSPIRE].

[52] A.E. Cárcamo Hernández, S. Kovalenko, J.W.F. Valle and C.A. Vaquera-Araujo, Predictive Pati-Salam theory of fermion masses and mixing, JHEP 07 (2017) 118 [arXiv: 1705.06320] [INSPIRE].

[53] N. Bernal, A.E. Cárcamo Hernández, I. de Medeiros Varzielas and S. Kovalenko, Fermion masses and mixings and dark matter constraints in a model with radiative seesaw mechanism, JHEP 05 (2018) 053 [arXiv: 1712.02792] [INSPIRE].

[54] A.E. Cárcamo Hernández, H.N. Long and V.V. Vien, Fermion masses and mixings in a 3-3-1 model with $\Delta(27)$ family symmetry and inverse seesaw mechanism, arXiv: 1803.01636 [INSPIRE].

[55] F.J. de Anda and S.F. King, An $S_{4} \times \mathrm{SU}(5)$ SUSY GUT of flavour in $6 d$, arXiv: 1803.04978 [INSPIRE].

[56] A.E. Cárcamo Hernández and S.F. King, Muon anomalies and the SU(5) Yukawa relations, arXiv: 1803.07367 [INSPIRE].

[57] G. Altarelli and F. Feruglio, Theoretical models of neutrino masses and mixings, Springer Tracts Mod. Phys. 190 (2003) 169 [hep-ph/0206077] [INSPIRE].

[58] G. Altarelli and F. Feruglio, Discrete Flavor Symmetries and Models of Neutrino Mixing, Rev. Mod. Phys. 82 (2010) 2701 [arXiv: 1002.0211] [InSPIRE]. 
[59] H. Ishimori, T. Kobayashi, H. Ohki, Y. Shimizu, H. Okada and M. Tanimoto, Non-Abelian Discrete Symmetries in Particle Physics, Prog. Theor. Phys. Suppl. 183 (2010) 1 [arXiv: 1003.3552] [INSPIRE].

[60] S.F. King and C. Luhn, Neutrino Mass and Mixing with Discrete Symmetry, Rept. Prog. Phys. 76 (2013) 056201 [arXiv: 1301.1340] [INSPIRE].

[61] S.F. King, A. Merle, S. Morisi, Y. Shimizu and M. Tanimoto, Neutrino Mass and Mixing: from Theory to Experiment, New J. Phys. 16 (2014) 045018 [arXiv:1402.4271] [InSPIRE].

[62] S.F. King, Unified Models of Neutrinos, Flavour and CP-violation, Prog. Part. Nucl. Phys. 94 (2017) 217 [arXiv: 1701.04413] [INSPIRE].

[63] H. Fritzsch, Calculating the Cabibbo Angle, Phys. Lett. B 70 (1977) 436.

[64] T. Fukuyama and H. Nishiura, Mass matrix of Majorana neutrinos, hep-ph/9702253 [INSPIRE].

[65] D.-s. Du and Z.-z. Xing, A Modified Fritzsch ansatz with additional first order perturbation, Phys. Rev. D 48 (1993) 2349 [InSPIRE].

[66] R. Barbieri, G.R. Dvali, A. Strumia, Z. Berezhiani and L.J. Hall, Flavor in supersymmetric grand unification: A Democratic approach, Nucl. Phys. B 432 (1994) 49 [hep-ph/9405428] [INSPIRE].

[67] R.D. Peccei and K. Wang, Natural mass matrices, Phys. Rev. D 53 (1996) 2712 [hep-ph/9509242] [INSPIRE].

[68] H. Fritzsch and Z.-z. Xing, Mass and flavor mixing schemes of quarks and leptons, Prog. Part. Nucl. Phys. 45 (2000) 1 [hep-ph/9912358] [INSPIRE].

[69] R.G. Roberts, A. Romanino, G.G. Ross and L. Velasco-Sevilla, Precision Test of a Fermion Mass Texture, Nucl. Phys. B 615 (2001) 358 [hep-ph/0104088] [INSPIRE].

[70] H. Nishiura, K. Matsuda, T. Kikuchi and T. Fukuyama, Phenomenological analysis of lepton and quark mass matrices, Phys. Rev. D 65 (2002) 097301 [hep-ph/0202189] [INSPIRE].

[71] I. de Medeiros Varzielas and G.G. Ross, SU(3) family symmetry and neutrino bi-tri-maximal mixing, Nucl. Phys. B 733 (2006) 31 [hep-ph/0507176] [INSPIRE].

[72] A.E. Carcamo Hernandez, R. Martinez and J.A. Rodriguez, Different kind of textures of Yukawa coupling matrices in the two Higgs doublet model type-III, Eur. Phys. J. C 50 (2007) 935 [hep-ph/0606190] [INSPIRE].

[73] Y. Kajiyama, M. Raidal and A. Strumia, The Golden ratio prediction for the solar neutrino mixing, Phys. Rev. D 76 (2007) 117301 [arXiv:0705.4559] [INSPIRE].

[74] A.E. Carcamo Hernandez and R. Rahman, A SUSY GUT model with pseudo-Goldstone Higgs doublets, Rev. Mex. Fis. 62 (2016) 100 [arXiv:1007.0447] [InSPIRE].

[75] G.C. Branco, D. Emmanuel-Costa and C. Simoes, Nearest-Neighbour Interaction from an Abelian Symmetry and Deviations from Hermiticity, Phys. Lett. B 690 (2010) 62 [arXiv: 1001.5065$]$ [INSPIRE].

[76] P. Leser and H. Pas, Neutrino mass hierarchy and the origin of leptonic flavor mixing from the righthanded sector, Phys. Rev. D 84 (2011) 017303 [arXiv:1104.2448] [INSPIRE].

[77] M. Gupta and G. Ahuja, Flavor mixings and textures of the fermion mass matrices, Int. J. Mod. Phys. A 27 (2012) 1230033 [arXiv:1302.4823] [INSPIRE]. 
[78] P.S.B. Dev and A. Pilaftsis, Minimal Radiative Neutrino Mass Mechanism for Inverse Seesaw Models, Phys. Rev. D 86 (2012) 113001 [arXiv:1209.4051] [INSPIRE].

[79] A.E. Carcamo Hernandez, C.O. Dib, N. Neill H and A.R. Zerwekh, Quark masses and mixings in the RS1 model with a condensing 4th generation, JHEP 02 (2012) 132 [arXiv:1201.0878] [INSPIRE].

[80] A.E. Carcamo Hernandez, R. Martinez and F. Ochoa, Radiative seesaw-type mechanism of quark masses in $\mathrm{SU}(3)_{C} \otimes \mathrm{SU}(3)_{L} \otimes \mathrm{U}(1)_{X}$, Phys. Rev. D 87 (2013) 075009 [arXiv:1302.1757] [INSPIRE].

[81] H. Päs and E. Schumacher, Leptogenesis and CP violation in $\mathrm{SU}(5)$ models with lepton flavor mixing originating from the right-handed sector, Phys. Rev. D 89 (2014) 096010 [arXiv: 1401.2328] [INSPIRE].

[82] A.E. Carcamo Hernandez, S. Kovalenko and I. Schmidt, An SU(5) grand unified model with discrete flavour symmetries, arXiv:1411.2913 [INSPIRE].

[83] A.E. Cárcamo Hernández and I. de Medeiros Varzielas, Viable textures for the fermion sector, J. Phys. G 42 (2015) 065002 [arXiv:1410.2481] [INSPIRE].

[84] H. Nishiura and T. Fukuyama, Simple neutrino mass matrix with only two free parameters, Mod. Phys. Lett. A 29 (2014) 0147 [arXiv: 1405.2416] [InSPIRE].

[85] M. Frank, C. Hamzaoui, N. Pourtolami and M. Toharia, Unified Flavor Symmetry from warped dimensions, Phys. Lett. B 742 (2015) 178 [arXiv:1406.2331] [INSPIRE].

[86] A. Ghosal and R. Samanta, Probing texture zeros with scaling ansatz in inverse seesaw, JHEP 05 (2015) 077 [arXiv:1501.00916] [INSPIRE].

[87] R. Sinha, R. Samanta and A. Ghosal, Maximal Zero Textures in Linear and Inverse Seesaw, Phys. Lett. B 759 (2016) 206 [arXiv: 1508.05227] [InSPIRE].

[88] H. Nishiura and T. Fukuyama, Simple mass matrices of neutrinos and quarks consistent with observed mixings and masses, Phys. Lett. B 753 (2016) 57 [arXiv:1510.01035] [INSPIRE].

[89] R. Samanta and A. Ghosal, Probing maximal zero textures with broken cyclic symmetry in inverse seesaw, Nucl. Phys. B 911 (2016) 846 [arXiv:1507.02582] [INSPIRE].

[90] R.R. Gautam, M. Singh and M. Gupta, Neutrino mass matrices with one texture zero and a vanishing neutrino mass, Phys. Rev. D 92 (2015) 013006 [arXiv:1506.04868] [INSPIRE].

[91] H. Päs and E. Schumacher, Common origin of $R_{K}$ and neutrino masses, Phys. Rev. D 92 (2015) 114025 [arXiv: 1510.08757] [inSPIRE].

[92] M. Gupta and G. Ahuja, Flavor mixings and textures of the fermion mass matrices, Int. J. Mod. Phys. A 27 (2012) 1230033 [arXiv:1302.4823] [INSPIRE].

[93] W. Wang and Z.-L. Han, Radiative linear seesaw model, dark matter and $\mathrm{U}(1)_{B-L}$, Phys. Rev. D 92 (2015) 095001 [arXiv: 1508.00706] [inSPIRE].

[94] G. von Gersdorff, Natural Fermion Hierarchies from Random Yukawa Couplings, JHEP 09 (2017) 094 [arXiv: 1705.05430] [INSPIRE].

[95] H. Georgi and A. Pais, Generalization of Gim: Horizontal and Vertical Flavor Mixing, Phys. Rev. D 19 (1979) 2746 [INSPIRE].

[96] J.W.F. Valle and M. Singer, Lepton Number Violation With Quasi Dirac Neutrinos, Phys. Rev. D 28 (1983) 540 [INSPIRE]. 
[97] F. Pisano and V. Pleitez, An SU(3) $\times \mathrm{U}(1)$ model for electroweak interactions, Phys. Rev. D 46 (1992) 410 [hep-ph/9206242] [INSPIRE].

[98] R. Foot, O.F. Hernandez, F. Pisano and V. Pleitez, Lepton masses in an $\mathrm{SU}(3)_{L} \times \mathrm{U}(1)_{N}$ gauge model, Phys. Rev. D 47 (1993) 4158 [hep-ph/9207264] [INSPIRE].

[99] P.H. Frampton, Chiral dilepton model and the flavor question, Phys. Rev. Lett. 69 (1992) 2889 [INSPIRE].

[100] H.N. Long, $\mathrm{SU}(3)_{L} \times \mathrm{U}(1)_{N}$ model for right-handed neutrino neutral currents, Phys. Rev. D 54 (1996) 4691 [hep-ph/9607439] [INSPIRE].

[101] H.N. Long, The 331 model with right handed neutrinos, Phys. Rev. D 53 (1996) 437 [hep-ph/9504274] [INSPIRE].

[102] R. Foot, H.N. Long and T.A. Tran, $\mathrm{SU}(3)_{L} \otimes \mathrm{U}(1)_{N}$ and $\mathrm{SU}(4)_{L} \otimes \mathrm{U}(1)_{N}$ gauge models with right-handed neutrinos, Phys. Rev. D 50 (1994) R34 [hep-ph/9402243] [INSPIRE].

[103] S.M. Boucenna, S. Morisi and A. Vicente, The LHC diphoton resonance from gauge symmetry, Phys. Rev. D 93 (2016) 115008 [arXiv: 1512.06878] [INSPIRE].

[104] A.E.C. Hernández and I. Nišandžić, LHC diphoton resonance at $750 \mathrm{GeV}$ as an indication of $\mathrm{SU}(3)_{L} \times \mathrm{U}(1)_{X}$ electroweak symmetry, Eur. Phys. J. C 76 (2016) 380 [arXiv: 1512.07165] [INSPIRE].

[105] R.M. Fonseca and M. Hirsch, A flipped 331 model, JHEP 08 (2016) 003 [arXiv: 1606.01109] [INSPIRE].

[106] C. Hati, S. Patra, M. Reig, J.W.F. Valle and C.A. Vaquera-Araujo, Towards gauge coupling unification in left-right symmetric $\mathrm{SU}(3)_{c} \times \mathrm{SU}(3)_{L} \times \mathrm{SU}(3)_{R} \times \mathrm{U}(1)_{X}$ theories, Phys. Rev. D 96 (2017) 015004 [arXiv: 1703.09647] [InSPIRE].

[107] C.A. de Sousa Pires and O.P. Ravinez, Charge quantization in a chiral bilepton gauge model, Phys. Rev. D 58 (1998) 035008 [hep-ph/9803409] [INSPIRE].

[108] P.V. Dong and H.N. Long, Electric charge quantization in $\mathrm{SU}(3)(C) \times \mathrm{SU}(3)(L) \times \mathrm{U}(1)(X)$ models, Int. J. Mod. Phys. A 21 (2006) 6677 [hep-ph/0507155] [INSPIRE].

[109] J.C. Montero, V. Pleitez and O. Ravinez, Soft superweak CP-violation in a 331 model, Phys. Rev. D 60 (1999) 076003 [hep-ph/9811280] [InSPIRE].

[110] J.C. Montero, C.C. Nishi, V. Pleitez, O. Ravinez and M.C. Rodriguez, Soft CP-violation in $K$ meson systems, Phys. Rev. D 73 (2006) 016003 [hep-ph/0511100] [INSPIRE].

[111] P.B. Pal, The Strong CP question in $\mathrm{SU}(3)(C) \times \mathrm{SU}(3)(L) \times \mathrm{U}(1)(N)$ models, Phys. Rev. D 52 (1995) 1659 [hep-ph/9411406] [INSPIRE].

[112] A.G. Dias, V. Pleitez and M.D. Tonasse, Naturally light invisible axion in models with large local discrete symmetries, Phys. Rev. D 67 (2003) 095008 [hep-ph/0211107] [INSPIRE].

[113] A.G. Dias and V. Pleitez, Stabilizing the invisible axion in 3-3-1 models, Phys. Rev. D 69 (2004) 077702 [hep-ph/0308037] [INSPIRE].

[114] A.G. Dias, C.A. de S. Pires and P.S. Rodrigues da Silva, Discrete symmetries, invisible axion and lepton number symmetry in an economic 331 model, Phys. Rev. D 68 (2003) 115009 [hep-ph/0309058] [INSPIRE].

[115] J.K. Mizukoshi, C.A. de S. Pires, F.S. Queiroz and P.S. Rodrigues da Silva, WIMPs in a 3-3-1 model with heavy Sterile neutrinos, Phys. Rev. D 83 (2011) 065024 [arXiv: 1010.4097] [INSPIRE]. 
[116] A.G. Dias, C.A. de S. Pires and P.S. Rodrigues da Silva, The Left-Right $\mathrm{SU}(3)(L) \times \mathrm{SU}(3)(R) \times \mathrm{U}(1)(X)$ Model with Light, keV and Heavy Neutrinos, Phys. Rev. D 82 (2010) 035013 [arXiv: 1003.3260] [INSPIRE].

[117] J.D. Ruiz-Alvarez, C.A. de S. Pires, F.S. Queiroz, D. Restrepo and P.S. Rodrigues da Silva, On the Connection of Gamma-Rays, Dark Matter and Higgs Searches at LHC, Phys. Rev. D 86 (2012) 075011 [arXiv:1206.5779] [INSPIRE].

[118] D. Cogollo, A.X. Gonzalez-Morales, F.S. Queiroz and P.R. Teles, Excluding the Light Dark Matter Window of a 331 Model Using LHC and Direct Dark Matter Detection Data, JCAP 11 (2014) 002 [arXiv: 1402.3271] [INSPIRE].

[119] P.S. Rodrigues da Silva, A Brief Review on WIMPs in 331 Electroweak Gauge Models, Phys. Int. 7 (2016) 15 [arXiv:1412.8633] [INSPIRE].

[120] T. Kitabayashi and M. Yasue, S(2L) permutation symmetry for left-handed mu and tau families and neutrino oscillations in an $\mathrm{SU}(3)_{L} \times \mathrm{SU}(1)_{N}$ gauge model, Phys. Rev. D 67 (2003) 015006 [hep-ph/0209294] [INSPIRE].

[121] S. Sen, Quark masses in supersymmetric $\mathrm{SU}(3)($ color $) \times \mathrm{SU}(3)(W) \times \mathrm{U}(1)(X)$ model with discrete T-prime flavor symmetry, Phys. Rev. D 76 (2007) 115020 [arXiv:0710.2734] [INSPIRE].

[122] P.V. Dong, H.N. Long, D.V. Soa and V.V. Vien, The 3-3-1 model with $S_{4}$ flavor symmetry, Eur. Phys. J. C 71 (2011) 1544 [arXiv: 1009.2328] [InSPIRE].

[123] P.V. Dong, H.N. Long, C.H. Nam and V.V. Vien, The $S_{3}$ flavor symmetry in 3-3-1 models, Phys. Rev. D 85 (2012) 053001 [arXiv:1111.6360] [INSPIRE].

[124] T. Kitabayashi and M. Yasue, Nearly bimaximal neutrino mixing in an $\mathrm{SU}(3)(L) \times \mathrm{U}(1)(N)$ gauge model with radiative neutrino masses, hep-ph/0006040 [INSPIRE].

[125] T. Kitabayashi and M. Yasue, Radiatively induced neutrino masses and oscillations in an $\mathrm{SU}(3)(L) \times \mathrm{U}(1)(N)$ gauge model, Phys. Rev. D 63 (2001) 095002 [hep-ph/0010087] [INSPIRE].

[126] T. Kitabayashi and M. Yasue, Large mixing angle $M S W$ solution in an $\mathrm{SU}(3)_{L} \times \mathrm{U}(1)_{N}$ gauge model with two loop radiative mechanism, Phys. Lett. B 508 (2001) 85 [hep-ph/0102228] [INSPIRE].

[127] D. Chang and H.N. Long, Interesting radiative patterns of neutrino mass in an $\mathrm{SU}(3)(C) \times \mathrm{SU}(3)(L) \times \mathrm{U}(1)(X)$ model with right-handed neutrinos, Phys. Rev. D 73 (2006) 053006 [hep-ph/0603098] [INSPIRE].

[128] P.V. Dong, D.T. Huong, T.T. Huong and H.N. Long, Fermion masses in the economical 3-3-1 model, Phys. Rev. D 74 (2006) 053003 [hep-ph/0607291] [InSPIRE].

[129] P.V. Dong and H.N. Long, Neutrino masses and lepton flavor violation in the 3-3-1 model with right-handed neutrinos, Phys. Rev. D 77 (2008) 057302 [arXiv:0801.4196] [INSPIRE].

[130] R. Martinez, F. Ochoa and P. Fonseca, A 3-3-1 Model with SU(8) Unification, arXiv: 1105.4623 [INSPIRE].

[131] D.T. Huong, L.T. Hue, M.C. Rodriguez and H.N. Long, Supersymmetric reduced minimal 3-3-1 model, Nucl. Phys. B 870 (2013) 293 [arXiv:1210.6776] [InSPIRE].

[132] S.M. Boucenna, S. Morisi and J.W.F. Valle, Radiative neutrino mass in 3-3-1 scheme, Phys. Rev. D 90 (2014) 013005 [arXiv: 1405.2332] [INSPIRE]. 
[133] H. Okada, N. Okada and Y. Orikasa, Radiative seesaw mechanism in a minimal 3-3-1 model, Phys. Rev. D 93 (2016) 073006 [arXiv:1504.01204] [INSPIRE].

[134] C.A. d.S. Pires, Neutrino mass mechanisms in 3-3-1 models: A short review, arXiv:1412.1002 [INSPIRE].

[135] M.E. Catano, R. Martinez and F. Ochoa, Neutrino masses in a 331 model with right-handed neutrinos without doubly charged Higgs bosons via inverse and double seesaw mechanisms, Phys. Rev. D 86 (2012) 073015 [arXiv:1206.1966] [inSPIRE].

[136] M. Reig, J.W.F. Valle and C.A. Vaquera-Araujo, Realistic $\mathrm{SU}(3)_{c} \otimes \mathrm{SU}(3)_{L} \otimes \mathrm{U}(1)_{X}$ model with a type-II Dirac neutrino seesaw mechanism, Phys. Rev. D 94 (2016) 033012 [arXiv: 1606.08499] [INSPIRE].

[137] A.E. Cárcamo Hernández, S. Kovalenko and I. Schmidt, Radiatively generated hierarchy of lepton and quark masses, JHEP 02 (2017) 125 [arXiv: 1611.09797] [INSPIRE].

[138] M. Singer, J.W.F. Valle and J. Schechter, Canonical Neutral Current Predictions From the Weak Electromagnetic Gauge Group SU(3) × U(1), Phys. Rev. D 22 (1980) 738 [inSPIRE].

[139] J.C. Montero, F. Pisano and V. Pleitez, Neutral currents and GIM mechanism in $\mathrm{SU}(3)_{L} \times \mathrm{U}(1)_{N}$ models for electroweak interactions, Phys. Rev. D 47 (1993) 2918 [hep-ph/9212271] [INSPIRE].

[140] L.A. Sanchez, W.A. Ponce and R. Martinez, $\mathrm{SU}(3)(c) \times \mathrm{SU}(3)(\ell) \times \mathrm{U}(1)(X)$ as an $E_{6}$ subgroup, Phys. Rev. D 64 (2001) 075013 [hep-ph/0103244] [INSPIRE].

[141] W.A. Ponce, J.B. Florez and L.A. Sanchez, Analysis of $\mathrm{SU}(3)(c) \times \mathrm{SU}(3)(L) \times \mathrm{U}(1)(X)$ local gauge theory, Int. J. Mod. Phys. A 17 (2002) 643 [hep-ph/0103100] [INSPIRE].

[142] W.A. Ponce, Y. Giraldo and L.A. Sanchez, Minimal scalar sector of 3-3-1 models without exotic electric charges, Phys. Rev. D 67 (2003) 075001 [hep-ph/0210026] [INSPIRE].

[143] R. Martinez and F. Ochoa, The Landau pole and Z-prime decays in the 331 bilepton model, Eur. Phys. J. C 51 (2007) 701 [hep-ph/0606173] [INSPIRE].

[144] L.T. Hue and L.D. Ninh, The simplest 3-3-1 model, Mod. Phys. Lett. A 31 (2016) 1650062 [arXiv: 1510.00302] [INSPIRE].

[145] R.A. Diaz, R. Martinez and F. Ochoa, $\mathrm{SU}(3)(c) \times \mathrm{SU}(3)(L) \times \mathrm{U}(1)(X)$ models for beta arbitrary and families with mirror fermions, Phys. Rev. D 72 (2005) 035018 [hep-ph/0411263] [INSPIRE].

[146] A.E. Carcamo Hernandez, R. Martinez and F. Ochoa, $Z$ and $Z$ ' decays with and without FCNC in 331 models, Phys. Rev. D 73 (2006) 035007 [hep-ph/0510421] [INSPIRE].

[147] H. Okada, N. Okada, Y. Orikasa and K. Yagyu, Higgs phenomenology in the minimal $\mathrm{SU}(3)_{L} \times \mathrm{U}(1)_{X}$ model, Phys. Rev. D 94 (2016) 015002 [arXiv: 1604.01948] [INSPIRE].

[148] C. Salazar, R.H. Benavides, W.A. Ponce and E. Rojas, LHC Constraints on 3-3-1 Models, JHEP 07 (2015) 096 [arXiv:1503.03519] [InSPIRE].

[149] R. Martinez and F. Ochoa, MasS-matrix ansatz and constraints on B0(s) - anti-B0(s) mixing in 331 models, Phys. Rev. D 77 (2008) 065012 [arXiv:0802.0309] [InSPIRE].

[150] A.J. Buras, F. De Fazio and J. Girrbach, 331 models facing new $b \rightarrow s \mu^{+} \mu^{-}$data, JHEP 02 (2014) 112 [arXiv:1311.6729] [INSPIRE].

[151] A.J. Buras, F. De Fazio and J. Girrbach-Noe, $Z$ - $Z^{\prime}$ mixing and $Z$-mediated FCNCs in $\mathrm{SU}(3)_{C} \times \mathrm{SU}(3)_{L} \times \mathrm{U}(1)_{X}$ models, JHEP 08 (2014) 039 [arXiv: 1405.3850] [INSPIRE]. 
[152] A.J. Buras, F. De Fazio, J. Girrbach and M.V. Carlucci, The Anatomy of Quark Flavour Observables in 331 Models in the Flavour Precision Era, JHEP 02 (2013) 023 [arXiv:1211.1237] [INSPIRE].

[153] E. Ponton, TASI 2011: Four Lectures on TeV Scale Extra Dimensions, in The Dark Secrets of the Terascale: Proceedings of TASI 2011, Boulder U.S.A. (2011), pg. 283 [arXiv: 1207.3827] [INSPIRE].

[154] M. Quirós, Higgs Bosons in Extra Dimensions, Mod. Phys. Lett. A 30 (2015) 1540012 [arXiv: 1311.2824] [INSPIRE].

[155] J.C. Montero, V. Pleitez and M.C. Rodriguez, A Supersymmetric 3-3-1 model, Phys. Rev. D 65 (2002) 035006 [hep-ph/0012178] [INSPIRE].

[156] M. Capdequi-Peyranere and M.C. Rodriguez, Charginos and neutralinos production at 3-3-1 supersymmetric model in e-e-scattering, Phys. Rev. D 65 (2002) 035001 [hep-ph/0103013] [INSPIRE].

[157] R.A. Diaz, R. Martinez and J.A. Rodriguez, A New supersymmetric $\mathrm{SU}(3)(L) \times \mathrm{U}(1)(X)$ gauge model, Phys. Lett. B 552 (2003) 287 [hep-ph/0208176] [INSPIRE].

[158] M.C. Rodriguez, Scalar sector in the minimal supersymmetric 3-3-1 model, Int. J. Mod. Phys. A 21 (2006) 4303 [hep-ph/0510333] [INSPIRE].

[159] S. Sen and A. Dixit, A Supersymmetric 3-3-1 model with MSSM-like scalar sector, hep-ph/0510393 [INSPIRE].

[160] S. Sen and A. Dixit, Higgs phenomenology in supersymmetric $\mathrm{SU}(3)(C) \times \mathrm{SU}(3)(L) \times \mathrm{U}(1)(X)$, hep-ph/0503078 [INSPIRE].

[161] P.V. Dong, D.T. Huong, M.C. Rodriguez and H.N. Long, Neutrino Masses in the Supersymmetric $\mathrm{SU}(3)(C) \times \mathrm{SU}(3)(L) \times \mathrm{U}(1)(X)$ Model with right-handed neutrinos, Eur. Phys. J. C 48 (2006) 229 [hep-ph/0604028] [INSPIRE].

[162] P.V. Dong, D.T. Huong, M.C. Rodriguez and H.N. Long, Supersymmetric economical 3-3-1 model, Nucl. Phys. B 772 (2007) 150 [hep-ph/0701137] [InSPIRE].

[163] D.T. Huong and H.N. Long, Neutralinos and charginos in supersymmetric economical 3-3-1 model, JHEP 07 (2008) 049 [arXiv:0804.3875] [INSPIRE].

[164] D.T. Huong and H.N. Long, Inflationary scenario in the supersymmetric economical 3-3-1 model, Phys. Atom. Nucl. 73 (2010) 791 [arXiv:0807.2346] [INSPIRE].

[165] D.T. Huong and H.N. Long, Non-thermal leptogenesis in supersymmetric 3-3-1 model with inflationary scenario, J. Phys. G 38 (2011) 015202 [arXiv:1004.1246] [INSPIRE].

[166] P.T. Giang, L.T. Hue, D.T. Huong and H.N. Long, Lepton-Flavor Violating Decays of Neutral Higgs to Muon and Tauon in Supersymmetric Economical 3-3-1 Model, Nucl. Phys. B 864 (2012) 85 [arXiv: 1204.2902] [INSPIRE].

[167] L.T. Hue, D.T. Huong and H.N. Long, Lepton flavor violating processes $\tau \rightarrow \mu \gamma, \tau \rightarrow 3 \mu$ and $Z \rightarrow \mu \tau$ in the Supersymmetric economical 3-3-1 model, Nucl. Phys. B 873 (2013) 207 [arXiv: 1301.4652] [INSPIRE].

[168] J.G. Ferreira, C.A. de S. Pires, P.S. Rodrigues da Silva and A. Sampieri, Higgs sector of the supersymmetric reduced 331 model, Phys. Rev. D 88 (2013) 105013 [arXiv:1308.0575] [INSPIRE]. 
[169] D.T. Binh, L.T. Hue, D.T. Huong and H.N. Long, Higgs revised in supersymmetric economical 3-3-1 model with B/ $\mu$-type terms, Eur. Phys. J. C 74 (2014) 2851 [arXiv: 1308.3085] [INSPIRE].

[170] J.G. Ferreira, C.A. de S. Pires, P.S. Rodrigues da Silva and C. Siqueira, On the Higgs-like boson in the Minimal Supersymmetric 3-3-1 Model, Eur. Phys. J. C 78 (2018) 225 [arXiv: 1706.04904] [INSPIRE].

[171] K. Bora, Updated values of running quark and lepton masses at GUT scale in SM, 2HDM and MSSM, Horizon 2 (2013) 112 [arXiv: 1206.5909] [INSPIRE].

[172] Z.-z. Xing, H. Zhang and S. Zhou, Updated Values of Running Quark and Lepton Masses, Phys. Rev. D 77 (2008) 113016 [arXiv:0712.1419] [INSPIRE].

[173] Particle Data Group collaboration, C. Patrignani et al., Review of Particle Physics, Chin. Phys. C 40 (2016) 100001.

[174] A. Das and N. Okada, Inverse seesaw neutrino signatures at the LHC and ILC, Phys. Rev. D 88 (2013) 113001 [arXiv:1207.3734] [InSPIRE].

[175] A. Das, P. Konar and S. Majhi, Production of Heavy neutrino in next-to-leading order QCD at the LHC and beyond, JHEP 06 (2016) 019 [arXiv: 1604.00608] [INSPIRE]. 